\title{
Skin Manifestations in COVID-19 Patients: Are They Indicators for Disease Severity? A Systematic Review
}

\author{
Parnian Jamshidi ${ }^{1}$, Bahareh Hajikhani ${ }^{2}$, Mehdi Mirsaeidi ${ }^{3 *}$, Hassan Vahidnezhad ${ }^{4}$, \\ Masoud Dadashi ${ }^{5}$ and Mohammad Javad Nasiri ${ }^{2 *}$ \\ ${ }^{1}$ Student Research Committee, School of Medicine, Shahid Beheshti University of Medical Sciences, Tehran, Iran, \\ ${ }^{2}$ Department of Microbiology, School of Medicine, Shahid Beheshti University of Medical Sciences, Tehran, Iran, ${ }^{3}$ Division of \\ Pulmonary and Critical Care, University of Miami, Miami, FL, United States, ${ }^{4}$ Department of Dermatology and Cutaneous \\ Biology, Sidney Kimmel Medical College, Thomas Jefferson University, Philadelphia, PA, United States, ${ }^{5}$ Department of \\ Microbiology, School of Medicine, Alborz University of Medical Sciences, Karaj, Iran
}

OPEN ACCESS

Edited by:

Zisis Kozlakidis, International Agency for Research on

Cancer (IARC), France

Reviewed by:

Dayi Zhang,

Tsinghua University, China

Sunny Oteikwu Ochigbo,

University of Calabar, Nigeria

*Correspondence:

Mohammad Javad Nasiri

mj.nasiri@hotmail.com

Mehdi Mirsaeidi

Msm249@med.miami.edu

Specialty section:

This article was submitted to Infectious Diseases - Surveillance,

Prevention and Treatment, a section of the journal

Frontiers in Medicine

Received: 27 November 2020

Accepted: 04 January 2021

Published: 16 February 2021

Citation:

Jamshidi P, Hajikhani B, Mirsaeidi M, Vahidnezhad H, Dadashi M and Nasiri MJ (2021) Skin Manifestations in COVID-19 Patients: Are They Indicators for Disease Severity? A Systematic Review.

Front. Med. 8:634208. doi: 10.3389/fmed.2021.634208
Introduction: Until now, there are several reports on cutaneous manifestations in COVID-19 patients. However, the link between skin manifestations and the severity of the disease remains debatable. We conducted a systematic review to evaluate the temporal relationship between different types of skin lesions and the severity of COVID-19.

Methods: A systematic search was conducted for relevant studies published between January and July 2020 using Pubmed/Medline, Embase, and Web of knowledge. The following keywords were used: "SARS-CoV-2" or "COVID-19" or "new coronavirus" or "Wuhan Coronavirus" or "coronavirus disease 2019" and "skin disease" or "skin manifestation" or "cutaneous manifestation."

Results: Out of 381 articles, 47 meet the inclusion criteria and a total of 1,847 patients with confirmed COVID-19 were examined. The overall frequency of cutaneous manifestations in COVID-19 patients was 5.95\%. The maculopapular rash was the main reported skin involvement (37.3\%) commonly occurred in middle-aged females with intermediate severity of the disease. Forty-eight percentage of the patients had a mild, 32\% a moderate, and 20\% a severe COVID-19 disease. The mild disease was mainly correlated with chilblain-like and urticaria-like lesions and patients with vascular lesions experienced a more severe disease. Seventy-two percentage of patients with chilblain-like lesions improved without any medication. The overall mortality rate was $4.5 \%$. Patients with vascular lesions had the highest mortality rate (18.2\%) and patients with urticaria-like lesions had the lowest mortality rate $(2.2 \%)$.

Conclusion: The mere occurrence of skin manifestations in COVID-19 patients is not an indicator for the disease severity, and it highly depends on the type of skin lesions. Chilblain-like and vascular lesions are the ends of a spectrum in which from chilblain-like to vascular lesions, the severity of the disease increases, and the patient's prognosis worsens. Those with vascular lesions should also be considered as high-priority patients for further medical care.

Keywords: COVID-19, coronavirus - COVID-19, skin manifestations, skin - pathology, systematic literature search, disease severity, mortality, prognosis 


\section{INTRODUCTION}

A viral outbreak caused by severe acute respiratory syndrome coronavirus 2 (SARS-CoV-2) emerged from Wuhan, China in late December 2019 (1). The disease was named coronavirus disease 2019 (COVID-19) by World Health Organization (WHO) and was declared as a pandemic on 11 March 2020 (2). After 1 year from the beginning of the pandemic, the full spectrum of COVID-19 presentations and its relationship with disease severity is still unknown. Fever, cough, chills, dyspnea, myalgia, and sore throat are the most common clinical presentations of COVID-19 and as time goes on, different other manifestations have been reported (3). Recently, skin lesions have been described as potential manifestations of COVID19 (4-6). The cutaneous changes reported to date include maculopapular rash, vesicular lesions, urticaria-like lesions, and chilblain-like lesions (4-8). Some of these skin manifestations arise before the signs and symptoms more commonly associated with COVID-19, suggesting that they could be presenting signs of COVID-19 (9). However, the link between skin manifestations and the severity of the disease remains debatable. Due to the great variety of reported dermatologic presentations as well as the inconsistency of data on the association between skin presentations of COVID-19 with poor outcome, we aimed to conduct a comprehensive systematic review on the clinical and histopathological characteristics of skin manifestations in relation to other features of confirmed COVID-19 patients and to evaluate the temporal relationship between different types of skin lesions and the severity of COVID-19.

\section{METHODS}

This review conforms to the "Preferred Reporting Items for Systematic Reviews and Meta-Analyses" (PRISMA) statement (10). Registration: PROSPERO (pending registration ID: 215422).

\section{Search Strategy and Selection Criteria}

To investigate the prevalence and characteristics of cutaneous manifestations in COVID-19 patients, a systematic search was conducted for relevant studies published between January and July 2020 using Pubmed, Embase, and Web of knowledge.

The following search terms were used (designed using $\mathrm{MeSH}$ keywords and Emtree terms): "SARS-CoV-2" or "COVID-19" or "new coronavirus" or "Wuhan Coronavirus" or "coronavirus disease 2019" and "skin disease" or "skin manifestation" or "cutaneous manifestation." Only studies included if they contained data about the skin manifestation in patients with confirmed COVID-19. There were no language restrictions. We got help from the Google Translate system for non-English papers. Review articles, duplicate publications, and articles with no relevant data were excluded from the analysis. Two authors independently screened the remaining articles. Finally, selected data were extracted from the full-texts of eligible publication by other investigators of the team.

\section{Data Extraction}

Data about the first author's name, date of publication, country, number of COVID-19 patients, number of cases with skin manifestations, age, gender, location and type of skin manifestations, associated cutaneous symptoms, the onset of skin lesions with systemic symptoms, the median duration of the lesions, treatment strategies and main histological findings of the lesions as well as comorbidities, associated symptoms, drug history, laboratory findings, severity and outcome of the patients were selected for further analysis. All cutaneous presentations related to COVID-19 were categorized into six groups: chilblain-like, vesicular, urticaria-like, maculopapular, vascular, and miscellaneous (lesions that we couldn't subscribe to any of the groups). Petechiae, purpura, livedo, and necrosis were classified into vascular lesions. Two authors (PJ, BH) independently extracted the data from the selected studies. The data was jointly reconciled, and disagreements were discussed and resolved between review authors (PJ, BH, MJN).

\section{Quality Assessment}

The critical appraisal checklist for case reports provided by the Joanna Briggs Institute (JBI) was used to perform a quality assessment of the studies (11).

\section{RESULTS}

At the first round of review, 381 articles were selected. After removing the duplicates and studies that did not meet the entry criteria, 88 full texts were finally selected for further assessment. Of these, only 47 articles had the characteristics appropriated for systematic review and were entered into the data extraction (Figure 1). Most of the studies were case reports $(47 \%, \mathrm{~N}: 22)$ followed by case series $(42.4 \%, \mathrm{~N}: 20)$, retrospective hospital/private section-based study $(6.4 \%, \mathrm{~N}: 3)$, and crosssectional $(4.2 \%, \mathrm{~N}: 2)$. Thirteen articles were originated from Italy, 11 from Spain, 10 from France, 5 from the USA, and others from Belgium, China, Thailand, Kuwait, Indonesia, Russia, Turkey, and Singapore. Information of the 47 analyzed articles can be found in Table $\mathbf{1}$.

A total of 1,847 patients with confirmed COVID-19 (based on positive RT-PCR or positive antibody tests) were examined in 47 articles, of which 597 patients had different skin manifestations. The overall frequency of cutaneous manifestations in COVID-19 patients was $5.95 \%$.

\section{Characteristics of the Cutaneous Lesions in Confirmed COVID-19 Patients}

The maculopapular rash was the main reported skin involvement (37.3\%) followed by chilblain-like lesions (18.4\%). The prevalence rate of vesicular and urticaria-like lesions was 15\% (Table 4).

The mean age of patients with cutaneous manifestations was 53.3 (ranging from 16 to 92) years. Chilblain-like lesions were more common in younger patients (mean age: 40.7 years) and vascular lesions were more common in the elderly (mean age: 72.3 years). 


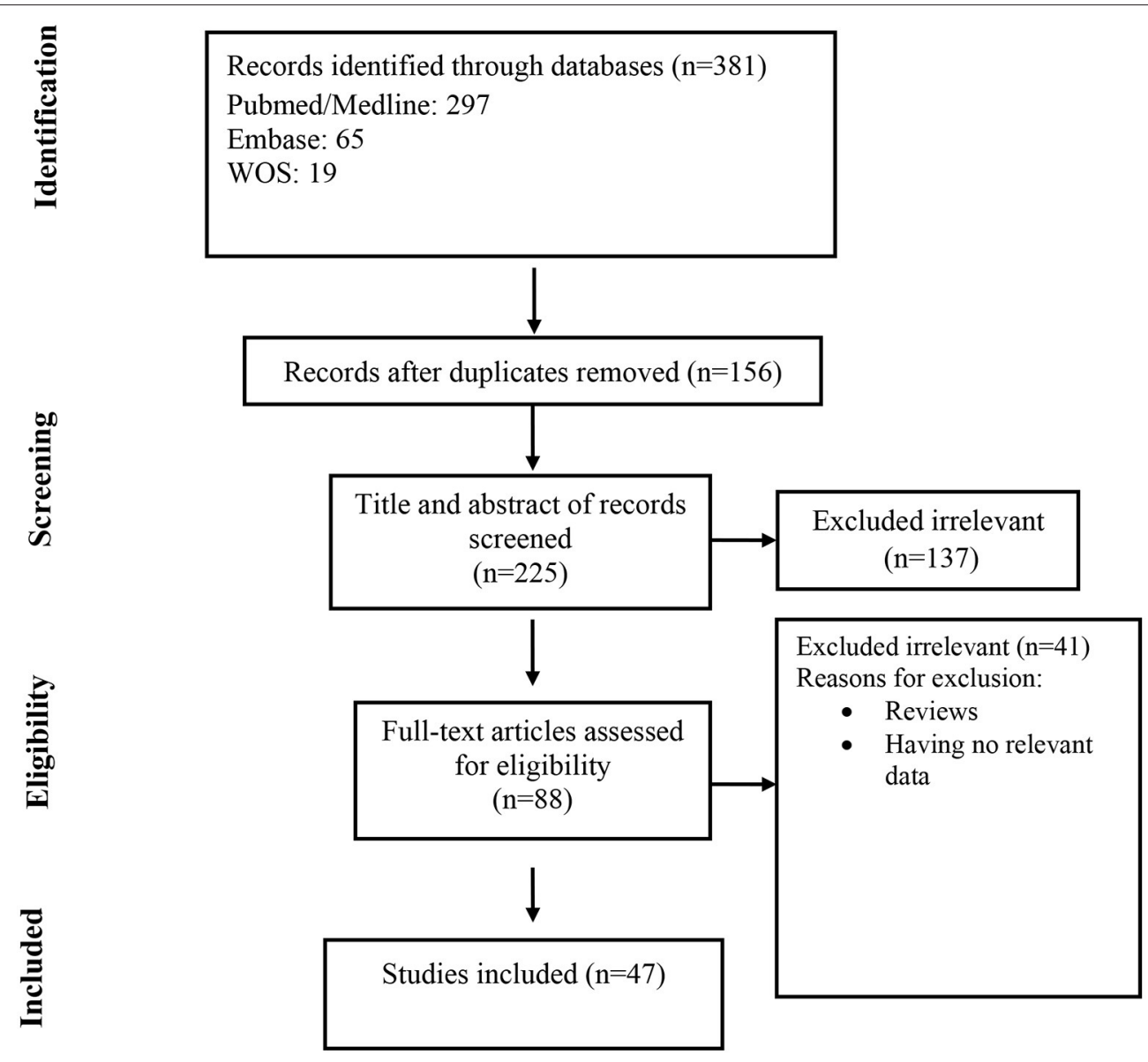

FIGURE 1 | Flow chart of study selection for inclusion in the systematic review and meta-analysis.

The prevalence of skin lesions was slightly higher in females than males (54 vs. $46 \%$ ). Urticaria-like, chilblain- like and miscellaneous lesions were more frequent among females (Table 4). Vascular lesions were more frequent in males (61\%). The prevalence of vesicular and maculopapular lesions was almost the same in men and women (51 and 49\%).

Trunk, lower limb, and upper limb were the main involved regions. Chilblain-like and vascular lesions were more common in acral areas and except for maculopapular lesions, others were commonly located in the trunk. The maculopapular lesions were more common in extremities. The involvement of palms and soles were rare. Mucous membrane involvement was reported in all types of skin lesions particularly maculopapular and vascular lesions, but it was not reported in chilblain-like lesions (Table 4). Vesicular rashes could have diffused polymorphic or localized monomorphic patterns $(27,37)$.

Out of 597, $397(66 \%)$ of the patients had associated cutaneous symptoms. Pruritus was the most prominent (238, $60 \%)$ particularly in vesicular lesions (89\%). Pain was the most frequent symptom in chilblain-like lesions (63.5\%) (see Table 4).

In the majority of patients (89.5\%), dermatologic manifestations presented after $(55 \%)$ or at the same time
(34.5\%) with the onset of systemic symptoms of COVID19. Urticaria-like lesions appeared usually as a concomitant symptom $(47 \%)$. In $3.5 \%$ of patients particularly with chilblainlike lesions, skin manifestations were the only presentation of COVID-19. In $7 \%$ of patients, skin manifestations occurred before the systemic symptoms, particularly in chilblain-like lesions (Table 4).

The median duration of skin lesions was about 9 days ranging from 1 to 18 days (Table 4). Urticaria-like lesions had the least duration (5 days) and chilblain-like lesions had the most duration (14 days).

No skin biopsy or histological examination of urticarialike lesions was performed. Therefore, the following results are related to other types of skin lesions.

Perivascular lymphocytic infiltration, spongiotic and interface dermatitis, and vacuolization or keratinocyte necrosis were the common histologic findings in skin biopsies, except for vesicular lesions. In vesicular lesions, the absence of inflammatory infiltrates, atrophic epidermis, and hyperkeratosis was reported. In almost all types of lesions (except maculopapular and vesicular lesions) thrombotic vasculopathy and red blood cell extravasation were present. Langerhans cell aggregations 
TABLE 1 | Characteristics of the included studies.

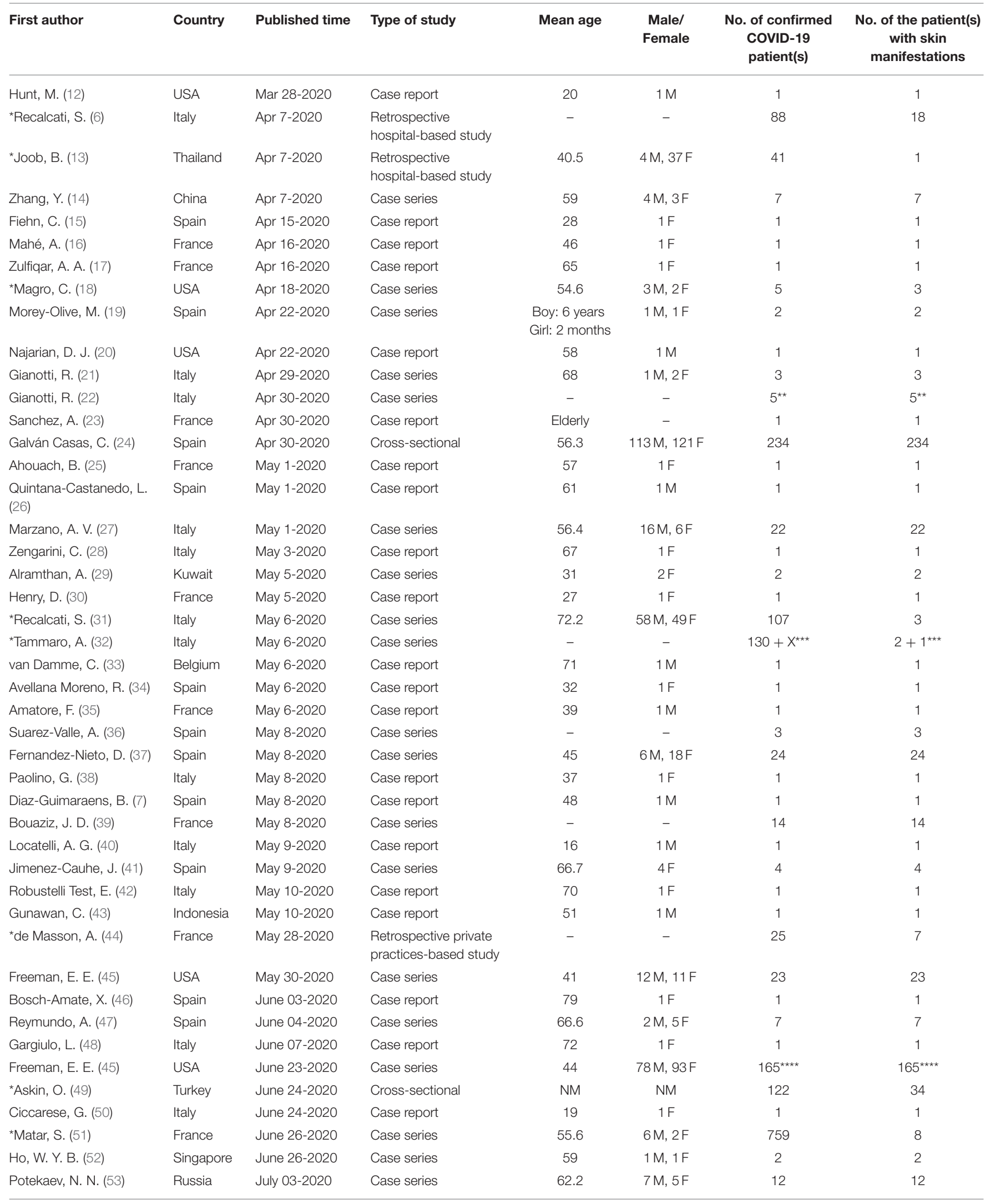


TABLE 1 | Continued

\begin{tabular}{|c|c|c|c|c|c|c|c|}
\hline First author & Country & Published time & Type of study & Mean age & $\begin{array}{c}\text { Male/ } \\
\text { Female }\end{array}$ & $\begin{array}{l}\text { No. of confirmed } \\
\text { CoVID-19 } \\
\text { patient(s) }\end{array}$ & $\begin{array}{c}\text { No. of the patient(s) } \\
\text { with skin } \\
\text { manifestations }\end{array}$ \\
\hline Le Cleach, L. (54) & France & July 06-2020 & Case series & 34 & $3 \mathrm{M}, 7 \mathrm{~F}$ & 10 & 10 \\
\hline Proietti, I. (55) & Italy & July 22-2020 & Case report & 6 months & $1 \mathrm{M}$ & 1 & 1 \\
\hline
\end{tabular}

*Articles that are included for calculating the prevalence of cutaneous manifestations in confirmed COVID-19 patients.

**Total population of cases was 8 but data of 5 patients were available only.

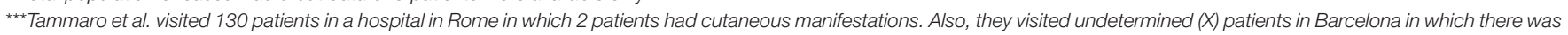
a patient with cutaneous manifestation. Note that for calculating the prevalence number we excluded the latter patient (because of the undetermined number of total case population).

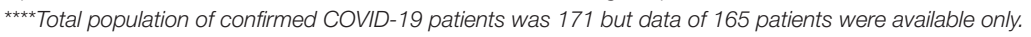

were seen within the epidermis in maculopapular lesions. Telangiectatic blood vessels were seen within the dermis of vascular and miscellaneous lesions. Virally-induced cytopathic alterations were absent according to reports on the miscellaneous category. Striking vascular and dermal deposits of complement factors (C5b-9, C3d, C4d) and IgM were present in four vascular rashes. Some studies performed an RT-PCR test on skin samples of maculopapular and vesicular lesions and the results were all negative for SARS-CoV-2. More details can be found in Table 2.

Most lesions required systemic corticosteroids (47\%) or had spontaneous remission (23.5\%). Antihistamines were the most widely used medication especially for urticaria-like lesions (57\%). Systemic corticosteroids were commonly used in vascular lesions (71\%) (Tables 2, 4).

\section{Characteristics of the Confirmed COVID-19 Patients With Skin Manifestations}

The overall prevalence of comorbidities among patients with skin manifestations was $17.9 \%$ (Table 4). Hypertension (39\%), diabetes (23\%), and dermatologic diseases (20\%) were the most frequent comorbidities, respectively. Utmost cases with comorbidity were across the patients with maculopapular lesions (40\%). Previous dermatologic illnesses were most common in patients with vesicular lesions (Table 4). Cardiovascular disease, hypertension, and obstructive lung diseases were common comorbidities amongst patients with vascular lesions (Table 4). Rheumatologic diseases were more frequent in patients with chilblain-like lesions (30\%). Diabetes was seen commonly in patients with urticaria-like lesions (46\%).

Fever (72\%), cough (61\%), fatigue/myalgia (51\%), and dyspnea (46\%) were the most common associated symptoms amongst the patients. Fever was more frequent in patients with vascular lesions (84\%) and less frequent in patients with chilblain-like lesions (39.5\%). Headache (41\%), dysosmia/hyposmia (27.5\%), nasal congestion/coryza (19\%), and irritability/confusion (10\%) were mainly seen in patients with vesicular lesions. Seventeen percentage of patients with chilblainlike lesions, $5 \%$ of patients with urticaria-like lesions, and $1 \%$ of patients with maculopapular lesions were asymptomatic. Bleeding presentations like epistaxis were seen just in patients with vascular lesions (Tables 3, 4).

Elevated D-dimer was the main laboratory finding in most of the cases, especially in patients with chilblain-like (100\%) and vascular (46\%) lesions. Disruption of coagulation condition (increase in PT, INR, and fibrinogen) was reported in patients with chilblain-like and vascular lesions (Tables 3, 4).

Regarding the drug history and medication regimen used for COVID-19, data of 389 out of 597 cases were available, most of which related to maculopapular and urticaria-like lesions. Fifty-two percentage of all cases and $72 \%$ of cases with chilblain-like lesions underwent symptomatic treatment with paracetamol, etc., or recovered without any medication. Chloroquine/hydroxychloroquine was the most common medication used in patients (45\%). Details in Tables 3, 4.

Most patients had mild disease (48\%). The majority of patients with chilblain-like lesions had mild disease (82\%) and the majority of patients with vascular lesions had severe disease $(68 \%)$. Also, most of the patients with maculopapular lesions were moderate $(43 \%)$ regarding severity (Tables 3,4 ).

The overall mortality rate among COVID-19 patients with cutaneous manifestations was $4.5 \%$. Patients with vascular lesions had the highest mortality rate $(18.2 \%)$ and patients with urticarialike lesions had the lowest mortality rate (2.2\%).

Details indicating characteristics of the lesions and the patients are shown in Tables $2-\mathbf{4}$.

\section{DISCUSSION}

After 1 year from the beginning of COVID-19 pandemic, the world is still facing a crisis. According to the current literature, more than half of the patients are asymptomatic leading to uncontrolled transmission of the virus (57-60). Recognizing COVID-19 related cutaneous manifestations may assist clinicians in early diagnosis of disease, before the development of respiratory symptoms, and may also be used to identify complications requiring treatment. The current study found that $10.5 \%$ of the COVID-19 patients reported skin lesions before the initiation of other symptoms or as their chief complaint. On the other hand, considering cutaneous manifestations is important to make the right diagnosis; as Joob et al. reported a COVID19 patient with petechiae misdiagnosed with dengue fever (13). Our data demonstrated that $34.5 \%$ of cutaneous manifestations occurred at the same time with other symptoms particularly urticaria-like lesions (47\%). It may suggest that urticaria-like lesions may be a diagnostic sign for COVID-19. The rest of the skin manifestations appeared later in the course of the disease 
TABLE 2 | Characteristics of the cutaneous lesions in confirmed COVID-19 patients.

\begin{tabular}{|c|c|c|c|c|c|c|c|c|}
\hline First author & Category & Location & Description & $\begin{array}{l}\text { Associated cutaneous } \\
\text { symptoms }\end{array}$ & $\begin{array}{l}\text { Rash onset with } \\
\text { other symptoms }\end{array}$ & $\begin{array}{l}\text { Median } \\
\text { duration }\end{array}$ & Main histologic findings & $\begin{array}{l}\text { Rash } \\
\text { treatment }\end{array}$ \\
\hline Locatelli, A. G. (40) & Chilblain-like & Dorsal aspects of the fingers & $\begin{array}{l}\text { Erythematous- } \\
\text { oedematous macules and } \\
\text { plaques (Chilblain-like) }\end{array}$ & Asymptomatic & After & - & $\begin{array}{l}\text { Oedema of the papillary } \\
\text { dermis, superficial and deep } \\
\text { lymphocytic infiltrate in a } \\
\text { peri-vascular and strong } \\
\text { peri-eccrine pattern; no signs } \\
\text { of endothelial damage. } \\
\text { consistent with a diagnosis of } \\
\text { chilblains }\end{array}$ & - \\
\hline Alramthan, A. (29) & Chilblain-like & $\begin{array}{l}\text { Dorsal aspect of fingers } \\
\text { bilaterally, subungual area of } \\
\text { the thumb }\end{array}$ & $\begin{array}{l}\text { Red-purple papules, } \\
\text { diffused erythema in the } \\
\text { subungual area }\end{array}$ & - & Chief complaint & - & - & - \\
\hline Suarez-Valle, A. (36) & Chilblain-like & $\begin{array}{l}\text { Toes (3), soles (1) (sparing } \\
\text { palms and mucous } \\
\text { membranes) }\end{array}$ & $\begin{array}{l}\text { Rounded reddish-purple } \\
\text { plaques, measuring } \\
\text { between } 0.5 \text { and } 1 \mathrm{~cm} \text {, } \\
\text { sharply defined, with no } \\
\text { retiform borders }\end{array}$ & - & 23 days after & 14 days & $\begin{array}{l}\text { Ischemic necrosis affecting } \\
\text { the epidermis and dermis with } \\
\text { signs of re-epithelialization. } \\
\text { vasculitis or microthrombi } \\
\text { were not found. }\end{array}$ & - \\
\hline de Masson, A. (44) & Chilblain-like & Hands and feet & Acral lesions (chilblains) & - & - & - & $\begin{array}{l}\text { Lichenoid dermatitis with a } \\
\text { perivascular and eccrine } \\
\text { mononuclear infiltrate, } \\
\text { vascular microthrombi }\end{array}$ & - \\
\hline Freeman, E. E. (45) & Chilblain-like & Hand (7), foot (20) & $\begin{array}{l}\text { Pernio-like acral skin } \\
\text { lesions }\end{array}$ & $\begin{array}{l}\text { Pruritus (8), Pain/Burning } \\
\text { (16) }\end{array}$ & $\begin{array}{l}\text { Before (4), After (11), } \\
\text { At the same time (3), } \\
\text { No other COVID-19 } \\
\text { symptoms (5) }\end{array}$ & 14 days & $\begin{array}{l}\text { Mild vacuolar interface } \\
\text { dermatitis with dense } \\
\text { superficial and deep } \\
\text { lymphocytic inflammation, } \\
\text { consistent with pernio vs. } \\
\text { connective tissue disease. No } \\
\text { thrombi were noted. }\end{array}$ & - \\
\hline Le Cleach, L. (54) & Chilblain-like & $\begin{array}{l}\text { Acral area of hand and foot, } \\
\text { dorsum of toes and soles, } \\
\text { lateral part of the foot }\end{array}$ & $\begin{array}{l}\text { Typical chilblains, severe } \\
\text { form with bullae, erythema } \\
\text { multiform-like lesions, } \\
\text { punctiform purpuric } \\
\text { lesions, diffuse vascular } \\
\text { erythema, and oedema }\end{array}$ & - & After/Before & - & $\begin{array}{l}\text { Vacuolization or apoptosis of } \\
\text { keratinocytes, superficial and } \\
\text { deep infiltrates mainly of } \\
\text { lymphocytes, perieccrine, and } \\
\text { perivascular reinforcement, } \\
\text { superficial capillary } \\
\text { thrombosis, dermal oedema }\end{array}$ & $\begin{array}{l}\text { Without } \\
\text { treatment, } \\
\text { topical } \\
\text { corticosteroids }\end{array}$ \\
\hline Najarian, D. J. (20) & Maculopapular & $\begin{array}{l}\text { Legs, thighs, forearms, arms, } \\
\text { shoulders, back, chest, and } \\
\text { abdomen (sparing the face, } \\
\text { hands, feet, and mucosa) }\end{array}$ & $\begin{array}{l}\text { Morbilliform erythematous } \\
\text { macules and patches }\end{array}$ & Pruritus & After & - & - & $\begin{array}{l}\text { Triamcinolone } \\
0.1 \%\end{array}$ \\
\hline
\end{tabular}


TABLE 2 | Continued

\begin{tabular}{|c|c|c|c|c|c|c|c|c|}
\hline First author & Category & Location & Description & $\begin{array}{l}\text { Associated cutaneous } \\
\text { symptoms }\end{array}$ & $\begin{array}{l}\text { Rash onset with } \\
\text { other symptoms }\end{array}$ & $\begin{array}{l}\text { Median } \\
\text { duration }\end{array}$ & Main histologic findings & $\begin{array}{l}\text { Rash } \\
\text { treatment }\end{array}$ \\
\hline Sanchez, A. (23) & Maculopapular & Trunk, back, thighs, arms & $\begin{array}{l}\text { Digitatepapulosquamous } \\
\text { eruption and } \\
\text { erythematous } \\
\text { periumbilical patch } \\
\text { (digitate scaly thin } \\
\text { plaques) }\end{array}$ & - & 7 days after & 7 days & $\begin{array}{l}\text { Spongiosis, parakeratosis, a } \\
\text { few rounded spongiotic } \\
\text { vesicles containing } \\
\text { aggregates of lymphocytes } \\
\text { and Langerhans cells. } \\
\text { moderate lymphohistiocytic } \\
\text { infiltration, negative COVID-19 } \\
\text { RT-PCR on a fresh skin } \\
\text { biopsy specimen }\end{array}$ & No \\
\hline Hunt, M. (12) & Maculopapular & $\begin{array}{l}\text { Trunk and extremities (sparing } \\
\text { the face, mucosal or ocular } \\
\text { involvement) }\end{array}$ & $\begin{array}{l}\text { Diffuse, morbilliform, } \\
\text { maculopapular (consistent } \\
\text { with a viral exanthem) }\end{array}$ & Non-pruritic & At the same time & - & - & - \\
\hline Ahouach, B. (25) & Maculopapular & Trunk, limbs & $\begin{array}{l}\text { Diffuse fixed erythematous } \\
\text { blanching maculopapular } \\
\text { lesions }\end{array}$ & Burning & - & - & $\begin{array}{l}\text { Spongiosis, basal cell } \\
\text { vacuolation and mild } \\
\text { perivascular lymphocytic } \\
\text { infiltrate, negative PCR on } \\
\text { whole-skin biopsy specimen } \\
\text { for SARS-CoV-2. }\end{array}$ & - \\
\hline $\begin{array}{l}\text { AvellanaMoreno, R. } \\
\text { (34) }\end{array}$ & Maculopapular & $\begin{array}{l}\text { Face, neck, thorax, abdomen, } \\
\text { buttocks, extremities including } \\
\text { folds and scalp, respecting } \\
\text { the palmoplantar region and } \\
\text { mucosa }\end{array}$ & $\begin{array}{l}\text { Generalized, pruritic } \\
\text { morbilliform rash } \\
\text { cephalocaudal progress } \\
\text { (petechial and } \\
\text { maculopapular on an } \\
\text { erythematous base), a } \\
\text { scaly reaction occurred on } \\
\text { the fourth day after the } \\
\text { rash started }\end{array}$ & Pruritus & 6 day after & 4 days & - & - \\
\hline Reymundo, A. (47) & Maculopapular & $\begin{array}{l}\text { Trunk (7), proximal upper } \\
\text { limbs (6), proximal lower limbs } \\
\text { (1) }\end{array}$ & - & - & - & - & $\begin{array}{l}\text { Mild superficial perivascular } \\
\text { lymphocytic infiltrate }\end{array}$ & $\begin{array}{l}\text { Without } \\
\text { treatment (1), } \\
\text { systemic } \\
\text { corticosteroid } \\
\text { (6) }\end{array}$ \\
\hline Morey-Olive, M. (19) & $\begin{array}{l}\text { Maculopapular (1), } \\
\text { Urticaria-like (1) }\end{array}$ & $\begin{array}{l}\text { Maculopapular: trunk, neck } \\
\text { spreading to the cheeks, } \\
\text { upper and lower extremities } \\
\text { (involving the palms) } \\
\text { Urticaria-like: face, upper } \\
\text { extremities spreading to the } \\
\text { trunk and lower extremities } \\
\text { (sparing palms and soles) }\end{array}$ & $\begin{array}{l}\text { Maculopapular: } \\
\text { erythematous, confluent, } \\
\text { non-pruritic } \\
\text { maculopapular exanthem } \\
\text { Urticaria-like: pruritic } \\
\text { Urticaria-like exanthem }\end{array}$ & Pruritus (1) & $\begin{array}{l}\text { After (1), At the } \\
\text { same time (1) }\end{array}$ & 5 days & - & No \\
\hline
\end{tabular}


TABLE 2 | Continued

\begin{tabular}{|c|c|c|c|c|c|c|c|c|}
\hline First author & Category & Location & Description & $\begin{array}{l}\text { Associated cutaneous } \\
\text { symptoms }\end{array}$ & $\begin{array}{l}\text { Rash onset with } \\
\text { other symptoms }\end{array}$ & $\begin{array}{l}\text { Median } \\
\text { duration }\end{array}$ & Main histologic findings & $\begin{array}{l}\text { Rash } \\
\text { treatment }\end{array}$ \\
\hline Gianotti, R. (22) & $\begin{array}{l}\text { Maculopapular (3), } \\
\text { Vascular (2) }\end{array}$ & Trunk, limb & $\begin{array}{l}\text { Maculopapular:diffusemacu } \\
\text { papulovesicular rash and } \\
\text { hemorrhagic dot-like area, } \\
\text { sligthlypapular } \\
\text { erythematous exanthema, } \\
\text { erythematous papular } \\
\text { eruption with crusted and } \\
\text { erosive lesions mimicking } \\
\text { Grover disease } \\
\text { Vascular: diffuse macular } \\
\text { livedoid } \\
\text { hemorrhagic lesions }\end{array}$ & & After (5) & - & $\begin{array}{l}\text { Maculopapular: classic } \\
\text { dyskeratotic cells, ballooning } \\
\text { multinucleated cells and } \\
\text { sparse necrotic keratinocytes } \\
\text { with lymphocytic satellitosis, } \\
\text { edematous dermis with many } \\
\text { eosinophils, Iymphocytic } \\
\text { vasculitis } \\
\text { Vascular: diffuse telangiectatic } \\
\text { small blood vessels in the } \\
\text { dermis, spongiotic dermatitis } \\
\text { with exocytosis along with a } \\
\text { large nest of Langerhans cells } \\
\text { and a dense perivascular } \\
\text { lymphocytic and eosinophilic } \\
\text { infiltration, lymphocytic } \\
\text { vasculitis, Intravascular } \\
\text { microthrombi in the small } \\
\text { dermal vessels. }\end{array}$ & - \\
\hline Mahé, A. (16) & Miscellaneous & $\begin{array}{l}\text { Both antecubital fossae } \\
\text { extended to the trunk and } \\
\text { axillary folds }\end{array}$ & Erythematous rash & - & 4 days after & 5 days & - & - \\
\hline $\begin{array}{l}\text { Robustelli Test, E. } \\
\text { (42) }\end{array}$ & Miscellaneous & $\begin{array}{l}\text { Face, trunk, upper and lower } \\
\text { limbs (sparing the mucous } \\
\text { membranes, palms, and } \\
\text { soles) }\end{array}$ & $\begin{array}{l}\text { Diffuse pustular eruption: } \\
\text { widespread eruption on } \\
\text { an erythematous- } \\
\text { oedematous base, with } \\
\text { scattered pinhead-sized } \\
\text { pustules and scales, } \\
\text { Targetoid lesions studded } \\
\text { with small pustules in a } \\
\text { symmetric pattern }\end{array}$ & Pruritus & After & - & $\begin{array}{l}\text { Subcorneal pustule with mild } \\
\text { focal acanthosis and } \\
\text { spongiosis, neutrophilic } \\
\text { exocytosis, sparse } \\
\text { keratinocyte necrosis, } \\
\text { perivascular lymphocytic } \\
\text { infiltrate with rare neutrophils } \\
\text { and eosinophils (consistent } \\
\text { with AGEP) }\end{array}$ & - \\
\hline Zengarini, C. (28) & Miscellaneous & $\begin{array}{l}\text { Neck, trunk, back, proximal } \\
\text { portions of limbs (sparing the } \\
\text { palmoplantar skin, face, and } \\
\text { mucous membranes) }\end{array}$ & $\begin{array}{l}\text { Erythematous confluent } \\
\text { rash, with undefined } \\
\text { margins, bleaching }\end{array}$ & Moderate pruritus & After & 7 days & $\begin{array}{l}\text { Slight superficial perivascular } \\
\text { lymphocytic infiltrate } \\
\text { extremely dilated vessel in the } \\
\text { papillary and mid dermis. }\end{array}$ & - \\
\hline
\end{tabular}




\begin{tabular}{|c|c|c|c|c|c|c|c|c|}
\hline First author & Category & Location & Description & $\begin{array}{l}\text { Associated cutaneous } \\
\text { symptoms }\end{array}$ & $\begin{array}{l}\text { Rash onset with } \\
\text { other symptoms }\end{array}$ & $\begin{array}{l}\text { Median } \\
\text { duration }\end{array}$ & Main histologic findings & $\begin{array}{l}\text { Rash } \\
\text { treatment }\end{array}$ \\
\hline Amatore, F. (35) & Miscellaneous & $\begin{array}{l}\text { Upper limbs, chest, neck, } \\
\text { abdomen, and palms (sparing } \\
\text { the face and mucous } \\
\text { membranes) }\end{array}$ & $\begin{array}{l}\text { Erythematous and } \\
\text { edematous annular fixed } \\
\text { plaques }\end{array}$ & Non-pruritic & At the same time & 7 days & $\begin{array}{l}\text { Predominantly superficial } \\
\text { perivascular infiltrate of } \\
\text { lymphocytes without } \\
\text { eosinophils, papillary dermal } \\
\text { edema, subtle epidermal } \\
\text { spongiosis, lichenoid, and } \\
\text { vacuolar interface dermatitis } \\
\text { with occasional dyskeratotic } \\
\text { keratinocytes in the basal } \\
\text { layer. No virally-induced } \\
\text { cytopathic alterations or } \\
\text { intranuclear inclusions were } \\
\text { present. Direct } \\
\text { immunofluorescence was } \\
\text { negative. }\end{array}$ & $\mathrm{HCQ}$ \\
\hline Gargiulo, L. (48) & Miscellaneous & Trunk, upper and lower limbs & $\begin{array}{l}\text { Erythema multiforme-like } \\
\text { (erythematous and slightly } \\
\text { edematous patches, } \\
\text { along with some isolated } \\
\text { typical target lesions) }\end{array}$ & Pruritus & 10 days Before & - & $\begin{array}{l}\text { Mixed perivascular and } \\
\text { interstitial infiltrate including } \\
\text { lymphocytes, granulocytes, } \\
\text { histiocytes, plasma cells, and } \\
\text { mast cells. }\end{array}$ & $\begin{array}{l}\text { Systemic } \\
\text { corticosteroid }\end{array}$ \\
\hline Ciccarese, G. (50) & Miscellaneous & $\begin{array}{l}\text { Lower limbs, Inner surface of } \\
\text { the lips, platelet, gingiva }\end{array}$ & $\begin{array}{l}\text { Cutaneous lesions: } \\
\text { erythematous macules, } \\
\text { papules, and petechiae } \\
\text { Oropharyngeal lesions: } \\
\text { erosions, ulcerations, } \\
\text { blood crusts, petechiae }\end{array}$ & Asymptomatic & 5 days after & 12 days & - & - \\
\hline Recalcati, S. (6) & $\begin{array}{l}\text { Miscellaneous (14), } \\
\text { Urticaria-like (3), } \\
\text { Vesiclular (1) }\end{array}$ & Trunk & $\begin{array}{l}\text { Erythematous rash (14), } \\
\text { widespread urticaria (3), } \\
\text { chickenpox-like vesicles } \\
\text { (1) }\end{array}$ & Low or absent pruritus & $\begin{array}{l}\text { At the same time (8), } \\
\text { After (10) }\end{array}$ & Few days & - & - \\
\hline Gianotti, R. (21) & Maculopapular (3) & Arms, trunk, lower limbs & $\begin{array}{l}\text { Widespread erythematous } \\
\text { macules, erythematous } \\
\text { crusted macules, and } \\
\text { papules }\end{array}$ & Pruritus (1) & After (2), Before (1) & $5,8,10$ days & $\begin{array}{l}\text { Perivascular dermatitis with } \\
\text { slight lymphocytic exocytosis } \\
\text { in a vasculitic pattern. } \\
\text { vascular thrombosis, Swollen } \\
\text { thrombosed vessels with } \\
\text { neutrophils, eosinophils and } \\
\text { nuclear debris, extravasated } \\
\text { red blood cells, focal } \\
\text { acantholyticsuprabasal clefts, } \\
\text { dyskeratotic and ballooning } \\
\text { herpes-like keratinocytes, } \\
\text { swollen vessels in the dermis } \\
\text { with dense lymphocyte } \\
\text { infiltration, mixed with rare } \\
\text { eosinophils. a nest of } \\
\text { Langerhans within the } \\
\text { epidermis. }\end{array}$ & No (3) \\
\hline
\end{tabular}




\begin{tabular}{|c|c|c|c|c|c|c|c|c|}
\hline First author & Category & Location & Description & $\begin{array}{l}\text { Associated cutaneous } \\
\text { symptoms }\end{array}$ & $\begin{array}{l}\text { Rash onset with } \\
\text { other symptoms }\end{array}$ & $\begin{array}{l}\text { Median } \\
\text { duration }\end{array}$ & Main histologic findings & $\begin{array}{l}\text { Rash } \\
\text { treatment }\end{array}$ \\
\hline $\begin{array}{l}\text { Jimenez-Cauhe, J. } \\
\text { (41) }\end{array}$ & Miscellaneous (4) & $\begin{array}{l}\text { Upper trunk, coalescing in the } \\
\text { back, and then spread to the } \\
\text { face and limbs within } 1 \text { week } \\
\text { (without the involvement of } \\
\text { palms and soles), platal } \\
\text { macules and petechiae (3) }\end{array}$ & $\begin{array}{l}\text { Erythematous papules } \\
\text { that progressively turned } \\
\text { to erythemato-violaceous } \\
\text { patches with a dusky } \\
\text { center, and a } \\
\text { pseudo-vesicle in the } \\
\text { middle, typical target } \\
\text { lesions (2). }\end{array}$ & - & 19.5 days after & 17.5 days & $\begin{array}{l}\text { Normal basket-weave stratum } \\
\text { corneum, mild to moderate } \\
\text { spongiosis in the epidermis, } \\
\text { dilated vessels filled with } \\
\text { neutrophils, extravasation of } \\
\text { red blood cells, lymphocytic } \\
\text { perivascular and interstitial } \\
\text { infiltrate, Basal vacuolar } \\
\text { changes with interface } \\
\text { dermatitis, lymphocytic } \\
\text { exocytosis }\end{array}$ & $\begin{array}{l}\text { Systemic } \\
\text { corticosteroids }\end{array}$ \\
\hline $\begin{array}{l}\text { Galván Casas, C. } \\
\text { (24) }\end{array}$ & $\begin{array}{l}\text { Chilblain-like (29), } \\
\text { Vesicular (17), } \\
\text { Urticaria-like (49), } \\
\text { Maculopapular } \\
\text { (122), Vascular (17) }\end{array}$ & $\begin{array}{l}\text { Chilblain-like: acral areas of } \\
\text { hands and feet. usually } \\
\text { asymmetrical } \\
\text { Vesicular: trunk, limbs } \\
\text { Urticaria-like: mostly trunk, a } \\
\text { few cases were palmar } \\
\text { Maculopapular: extremities, } \\
\text { mostly dorsum of the hands } \\
\text { Livedo/necrosis: trunk, } \\
\text { acral area }\end{array}$ & $\begin{array}{l}\text { Pseudo-chilblain (29), } \\
\text { Vesicular (17), Urticarial } \\
\text { (49), Maculopapules (122), } \\
\text { Livedo/necrosis (17) }\end{array}$ & - & $\begin{array}{l}\text { Before (9), At the } \\
\text { same time (147), } \\
\text { After (77) }\end{array}$ & $\begin{array}{l}\text { Chilblain-like: } \\
12.7 \text { days } \\
\text { Vesicular: } 10.4 \\
\text { days } \\
\text { Urticaria-like: } \\
6.8 \text { days } \\
\text { Maculopapular: } \\
8.6 \text { days }\end{array}$ & - & - \\
\hline Fiehn, C. (15) & Urticaria-like & Both heels & $\begin{array}{l}\text { Confluent } \\
\text { erythematous-yellowish } \\
\text { papules and plaques }\end{array}$ & Pruritus & 13 days After & - & - & - \\
\hline Gunawan, C. (43) & Urticaria-like & Face & Urticaria & Pruritus & 5 days After & 1 & & Loratadine \\
\hline $\begin{array}{l}\text { Quintana- } \\
\text { Castanedo, L. } \\
\text { (26) }\end{array}$ & Urticaria-like & $\begin{array}{l}\text { Thighs, arms, and forearms } \\
\text { (sparing the palms and soles) }\end{array}$ & $\begin{array}{l}\text { Urticarial rash consisting } \\
\text { of confluent, edematous, } \\
\text { and erythematous papules }\end{array}$ & Mild pruritus & Chief complaint & 7 days & - & Antihistamine \\
\hline Henry, D. (30) & Urticaria-like & Face, acral area, palm & $\begin{array}{l}\text { Disseminated } \\
\text { erythematous plaques } \\
\text { (Urticaria), papules in palm }\end{array}$ & Pruritus & Before & - & - & Antihistamine \\
\hline van Damme, C. (33) & Urticaria-like & - & $\begin{array}{l}\text { Extensive acute urticarial } \\
\text { rash }\end{array}$ & - & At the same time & - & - & Bilastine \\
\hline Paolino, G. (38) & Urticaria-like & Trunk, neck, face, lower limbs & $\begin{array}{l}\text { An urticaria-like lesion with } \\
\text { craniocaudal development }\end{array}$ & Non-pruritic & 3 days after & 8 days & - & - \\
\hline Proietti, I. (55) & Urticaria-like & Trunk, limbs & $\begin{array}{l}\text { Giant urticaria with } \\
\text { multiple lesions }\end{array}$ & - & $\begin{array}{l}14 \text { days after } \\
\text { RT-PCR test (no } \\
\text { associated } \\
\text { symptoms) }\end{array}$ & - & - & $\begin{array}{l}\text { Oral } \\
\text { betamethasone }\end{array}$ \\
\hline Zulfiqar, A. A. (17) & Vascular & Lower extremity & Purpura & - & 5 days after & 13 days & - & $\begin{array}{l}\text { IVIG, } \\
\text { Prednisolone, } \\
\text { Platelet } \\
\text { transfusion }\end{array}$ \\
\hline Joob, B. (13) & Vascular & - & Petechiae & - & - & - & - & No \\
\hline
\end{tabular}


TABLE 2 | Continued

\begin{tabular}{|c|c|c|c|c|c|c|c|c|}
\hline First author & Category & Location & Description & $\begin{array}{l}\text { Associated cutaneous } \\
\text { symptoms }\end{array}$ & $\begin{array}{l}\text { Rash onset with } \\
\text { other symptoms }\end{array}$ & $\begin{array}{l}\text { Median } \\
\text { duration }\end{array}$ & Main histologic findings & $\begin{array}{l}\text { Rash } \\
\text { treatment }\end{array}$ \\
\hline $\begin{array}{l}\text { Diaz-Guimaraens, B. } \\
\text { (7) }\end{array}$ & Vascular & $\begin{array}{l}\text { Symmetric periflexural } \\
\text { distribution: buttocks, } \\
\text { popliteal fossae, proximal } \\
\text { anterior thighs, and lower } \\
\text { abdomen (sparing the crural } \\
\text { folds and mucosa) }\end{array}$ & $\begin{array}{l}\text { Confluent erythematous } \\
\text { macules, papules, and } \\
\text { petechiae }\end{array}$ & Slightly pruritic & 3 days after & 5 days & $\begin{array}{l}\text { Perivascular lymphocytic } \\
\text { infiltrate, red cell } \\
\text { extravasation, and focal } \\
\text { papillary edema, along with } \\
\text { focal parakeratosis and } \\
\text { isolated dyskeratotic cells. No } \\
\text { features of thrombotic } \\
\text { vasculopathy were present }\end{array}$ & $\begin{array}{l}0.05 \% \\
\text { Betamethasor } \\
\text { dipropionate } \\
\text { cream, } \\
\text { Loratadine }\end{array}$ \\
\hline Magro, C. (18) & Vascular & $\begin{array}{l}\text { Buttocks, palms and soles, } \\
\text { chest, legs, and arms }\end{array}$ & $\begin{array}{l}\text { Purpuric reticulated } \\
\text { eruptions with } \\
\text { surrounding inflammation } \\
\text { (Livedoracemosa) }\end{array}$ & - & 4 days after & - & $\begin{array}{l}\text { Thrombogenic vasculopathy, } \\
\text { extensive necrosis of the } \\
\text { epidermis and adnexal } \\
\text { structures, interstitial and } \\
\text { perivascular neutrophilia with } \\
\text { prominent leukocytoclasia, } \\
\text { superficial vascular ectasia, } \\
\text { perivascular lymphocytic } \\
\text { infiltration, absence of clear } \\
\text { vasculitis, Significant vascular } \\
\text { deposits of C5b-9, C3d, and } \\
\text { C4d (in all cases) }\end{array}$ & - \\
\hline Recalcati, S. (6) & Vascular & Acral area, foot & $\begin{array}{l}\text { Acrocyanosis (2), foot } \\
\text { thrombosis (1) }\end{array}$ & - & - & - & - & - \\
\hline Zhang, Y (14) & Vascular & Finger/toe & $\begin{array}{l}\text { Acro-ischemia including } \\
\text { cyanosis, bulla, and dry } \\
\text { gangrene }\end{array}$ & - & - & $\begin{array}{l}12 \text { days to } \\
\text { death }\end{array}$ & - & - \\
\hline $\begin{array}{l}\text { Bosch-Amate, X. } \\
\text { (46) }\end{array}$ & Vascular & Both legs & $\begin{array}{l}\text { Retiform } \\
\text { purpuric-violaceous } \\
\text { patches of } 15 \mathrm{~cm} \text { with } \\
\text { some hemorrhagic blisters } \\
\text { and crusts suggestive of } \\
\text { retiform purpura }\end{array}$ & Pain & - & - & $\begin{array}{l}\text { Multiple thrombi occluding } \\
\text { most small-sized vessels of } \\
\text { the superficial and } \\
\text { mid-dermis, deposition of } \\
\text { IgM, C3, C9, and fibrinogen } \\
\text { within superficial-to-deep } \\
\text { dermal blood vessel walls. }\end{array}$ & - \\
\hline Bouaziz, J. D. (39) & $\begin{array}{l}\text { Vesicular (2), } \\
\text { Urticaria-like (1), } \\
\text { Chilblain-like (2), } \\
\text { Vascular (3), } \\
\text { Miscellaneous (6) }\end{array}$ & & $\begin{array}{l}\text { Inflammatory lesions were } \\
\text { reported in } 7 \text { patients: } \\
\text { exanthema (4), } \\
\text { chickenpox like vesicles } \\
\text { (2), cold urticaria (1), } \\
\text { Vascular lesions were } \\
\text { reported in } 7 \text { patients: } \\
\text { violaceous macules with } \\
\text { "porcelain-like" } \\
\text { appearance (1), livedo (1), } \\
\text { nonnecroticpurpura (1), } \\
\text { necrotic purpura (1), } \\
\text { chilblain appearance with } \\
\text { Raynaud's phenomenon } \\
\text { (1), chilblain (1), eruptive } \\
\text { cherry angioma (1). }\end{array}$ & - & A few days after & - & - & - \\
\hline
\end{tabular}


TABLE 2 | Continued

\begin{tabular}{|c|c|c|c|c|c|c|c|c|}
\hline First author & Category & Location & Description & $\begin{array}{l}\text { Associated cutaneous } \\
\text { symptoms }\end{array}$ & $\begin{array}{l}\text { Rash onset with } \\
\text { other symptoms }\end{array}$ & $\begin{array}{l}\text { Median } \\
\text { duration }\end{array}$ & Main histologic findings & $\begin{array}{l}\text { Rash } \\
\text { treatment }\end{array}$ \\
\hline Marzano, A. V. (27) & Vesicular (22) & Trunk (22), limbs (4) & $\begin{array}{l}\text { Scattered (16), diffuse (6), } \\
\text { Predominance of vesicles } \\
\text { (12), varicella-like } \\
\text { exanthem }\end{array}$ & Mild pruritus (9) & 3 days after & 8 days & $\begin{array}{l}\text { Basket-wave hyperkeratosis, } \\
\text { absence of inflammatory } \\
\text { infiltrate, atrophic epidermis, } \\
\text { vacuolar alteration with } \\
\text { disorganized keratinocytes } \\
\text { lacking orderly maturation, } \\
\text { enlarged and multinucleate } \\
\text { keratinocytes with } \\
\text { dyskeratotic (apoptotic) cells. }\end{array}$ & - \\
\hline $\begin{array}{l}\text { Fernandez-Nieto, D. } \\
\text { (37) }\end{array}$ & Vesicular (24) & $\begin{array}{l}\text { Head (4), anterior trunk (21), } \\
\text { posterior trunk (14), arms (8), } \\
\text { legs (10), palms-soles (2) }\end{array}$ & $\begin{array}{l}18 \text { disseminated pattern } \\
\text { (small papules, vesicles, } \\
\text { and pustules with varying } \\
\text { sizes of up to } 7-8 \mathrm{~mm} \\
\text { diameter, different stages } \\
\text { of the lesions appeared } \\
\text { simultaneously), } 6 \\
\text { localized pattern } \\
\text { (monomorphic lesions, of } \\
\text { up to } 3-4 \mathrm{~mm} \text { diameter, at } \\
\text { the same stage of } \\
\text { evolution, mostly trunk } \\
\text { involvement) }\end{array}$ & $\begin{array}{l}\text { Pruritus (20), } \\
\text { Asymptomatic (4) }\end{array}$ & $\begin{array}{l}\text { Before (2), At the } \\
\text { same time (3), After } \\
\text { (19) }\end{array}$ & 10 days & $\begin{array}{l}\text { Intraepidermal vesicles with } \\
\text { mild acantolisis and ballooned } \\
\text { keratinocytes consistent with } \\
\text { a viral infection, negative } \\
\text { SARS-CoV-2 RT-PCR on fluid } \\
\text { content of the vesicles }\end{array}$ & - \\
\hline Tammaro, A. (32) & Vesicular & Trunk, back & $\begin{array}{l}\text { Isolated herpetiform } \\
\text { lesions: lesions were } \\
\text { characterized by vesicles } \\
\text { surrounded by } \\
\text { erythematous halos. In } \\
\text { one of the patients, the } \\
\text { vesicles had started to } \\
\text { form crusts, numerous } \\
\text { vesicular isolated lesions } \\
\text { on her back. }\end{array}$ & Mild pruritus & After & - & - & - \\
\hline
\end{tabular}




\begin{tabular}{|c|c|c|c|c|c|c|c|c|}
\hline First author & Category & Location & Description & $\begin{array}{l}\text { Associated cutaneous } \\
\text { symptoms }\end{array}$ & $\begin{array}{l}\text { Rash onset with } \\
\text { other symptoms }\end{array}$ & $\begin{array}{l}\text { Median } \\
\text { duration }\end{array}$ & Main histologic findings & $\begin{array}{l}\text { Rash } \\
\text { treatment }\end{array}$ \\
\hline Potekaev, N. N. (53) & $\begin{array}{l}\text { Chilblain-like (1), } \\
\text { Vesicular (2), } \\
\text { Urticaria-like (1), } \\
\text { Maculopapular (4), } \\
\text { Vascular (4) }\end{array}$ & $\begin{array}{l}\text { Lower limb (6), upper limb (5), } \\
\text { trunk (4), first MTP joints (1), } \\
\text { ankles and dorsal surfaces of } \\
\text { the feet and toes (1) }\end{array}$ & $\begin{array}{l}\text { Vascular: papulonecrotic } \\
\text { rash with hemorrhagic } \\
\text { crusts, polymorphic } \\
\text { cutaneous vasculitis, } \\
\text { dense petechial and } \\
\text { ecchymotic rash } \\
\text { Chilblain-like: hyperemic } \\
\text { pernio-like lesions } \\
\text { Maculopapular: spotted } \\
\text { elements of bright pink } \\
\text { color, papulosquamous } \\
\text { rash (pytriasis rosea-like, } \\
\text { absence of the herald } \\
\text { patch), disseminated } \\
\text { pink-red maculopapular } \\
\text { rash resembling that of } \\
\text { measles, large bright red } \\
\text { foci } \\
\text { Vesicular: papulovesicular } \\
\text { eruptions with } \\
\text { surface erosions }\end{array}$ & Pain (1), Pruritus (1) & $\begin{array}{l}\text { Before (1), At the } \\
\text { same time (1), After } \\
\text { (5) }\end{array}$ & - & - & $\begin{array}{l}\text { Without } \\
\text { treatment (1), } \\
\text { Systemic } \\
\text { corticosteroid } \\
\text { (3) }\end{array}$ \\
\hline Freeman, E. E. (56) & $\begin{array}{l}\text { Chilblain-like (31), } \\
\text { Vesicular (18), } \\
\text { Urticaria-like (27), } \\
\text { Maculopapular (78), } \\
\text { Vascular (11) }\end{array}$ & $\begin{array}{l}\text { Hand (38), foot (51), face (32), } \\
\text { head (11), neck (26), chest } \\
\text { (49), abdomen (63), back (62), } \\
\text { arm (66), genitals (7), } \\
\text { leg/buttocks (72), entire body } \\
\text { (9) }\end{array}$ & $\begin{array}{l}\text { Maculopapular: } \\
\text { morbilliform rash, macular } \\
\text { erythema, } \\
\text { papulosquamous } \\
\text { Vascular: retiform purpura }\end{array}$ & $\begin{array}{l}\text { Pruritus (97), Burning/pain } \\
\text { (55) }\end{array}$ & $\begin{array}{l}\text { Before (17), After } \\
\text { (107), At the same } \\
\text { time (29), Chief } \\
\text { complaint (10) }\end{array}$ & - & $\begin{array}{l}\text { Vascular: thrombotic } \\
\text { vasculopathy, leukocytoclastic } \\
\text { vasculitis } \\
\text { Maculopapular: spongiosis } \\
\text { and dermal inflammation } \\
\text { Chilblain-like: vacuolar } \\
\text { interface dermatitis, } \\
\text { subepidermal edema, and } \\
\text { superficial and deep } \\
\text { lymphocytic inflammation } \\
\text { Miscellaneous (actually } \\
\text { distributed petechial, macular, } \\
\text { and urticarial eruption): } \\
\text { numerous dyskeratotic } \\
\text { keratinocytes, sparse } \\
\text { perivascular lymphohistiocytic } \\
\text { inflammation, and rare } \\
\text { dermal eosinophils. }\end{array}$ & - \\
\hline Matar, S. (51) & $\begin{array}{l}\text { Maculopapular, } \\
\text { Vesicular, } \\
\text { Miscellaneous }\end{array}$ & - & $\begin{array}{l}\text { Disseminated } \\
\text { maculopapular } \\
\text { exanthema, digitate } \\
\text { papulosquamous rash, } \\
\text { herpes recurrence, } \\
\text { papulovesicular rash, } \\
\text { Grover's disease }\end{array}$ & - & 13 days after & - & - & - \\
\hline
\end{tabular}




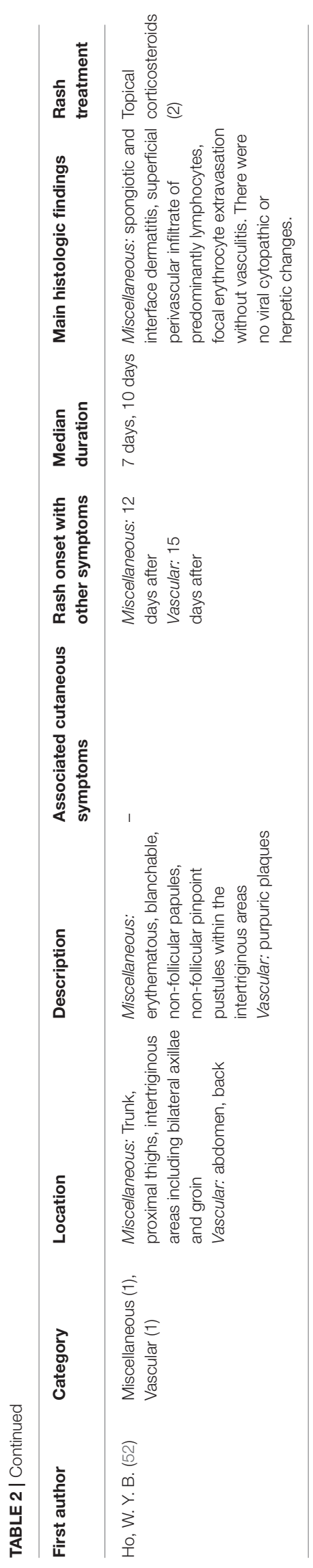

and mainly after the initiation of systemic symptoms (55\%) in our review. Galván Casas et al. suggested the chilblain-like and vesicular lesions as epidemiological markers for the disease (24). However, in our study, vesicular lesions (74\%) were the most important cutaneous manifestations usually appearing after systemic symptoms of the disease.

Most of the patients with skin manifestations were middleaged females, while, patients with chilblain-like lesions were younger (mean age: 40.7 years) and patients with vascular lesions were older individuals (mean age: 72.3 years). These findings are along with other studies about the chilblain-like lesions $(6,19,24$, 40). Maculopapular lesions were the most common dermatologic presentation of COVID-19 patients that commonly appeared at extremities. It occurred most often in middle-aged patients and was associated with moderate COVID-19 severity.

The overall mortality rate between the COVID-19 patients with skin presentations was $4.5 \%$, with the point that there was the lowest mortality rate among the patients with urticaria-like lesions (2.2\%) and contradictory, there was the highest mortality rate among the patients with vascular lesions (18.2\%). Previous studies showed a pooled mortality rate of $3.2-6 \%$ in patients with COVID-19 $(61,62)$. Thus, the mortality rate of COVID-19 patients with skin manifestations is proportionate to the overall mortality rate of the disease.

Regardless of the type of skin lesions, $80 \%$ of COVID-19 patients with cutaneous manifestations experienced a mild and moderate, and 20\% a severe COVID-19 disease. A previous study from the Chinese Center for Disease Control and Prevention reported that $81 \%$ of COVID-19 patients had a mild, $14 \%$ a severe, and $5 \%$ a critical disease (63). We don't have any specific data on patients without skin manifestations but comparing the COVID-19 severity in patients with skin manifestations and COVID-19 patients, regardless of their symptoms, demonstrates no obvious difference. Future cohort studies are required to compare the disease severity and outcome of COVID-19 patients with and without skin manifestations.

There is a wide range of cutaneous manifestations related to COVID-19 that in terms of age, associated symptoms, comorbidity, medication, severity, and mortality, chilblainlike lesions, and vascular lesions are the ends of this spectrum. Chilblain-like, urticaria-like, vesicular, maculopapular, miscellaneous, and vascular lesions are associated with an increase in COVID-19 severity and worsening the prognosis, respectively. Vascular lesions were more prevalent in males (61\%) compared to females (39\%). Considering the more severe disease and higher mortality rate in patients with vascular lesions, we can conclude that COVID-19 is more severe in males compared to females. This finding is compatible with our recent article, in which we assessed the sex-specific risk of mortality in COVID-19 patients (62).

Up to date, there is conflicting information about the potential possibility of transmitting the virus through the skin $(37,40,64)$. Further investigations are required to identify the pathophysiology of SARS-COV-2 and to determine whether patients with long-lasting skin lesions (e.g., chilblain-like lesions) are capable of infecting other individuals through skin contact or not. 
TABLE 3 | Characteristics of the confirmed COVID-19 patients with skin manifestations.

\begin{tabular}{|c|c|c|c|c|c|}
\hline First author & Comorbidity & Associated symptoms & Drug history & Laboratory findings & Severity/outcome \\
\hline Locatelli, A. G. (40) & - & Dysgeusia, mild diarrhea & - & - & Non-severe \\
\hline Alramthan, A. (29) & - & Asymptomatic & - & - & - \\
\hline Suarez-Valle A. (36) & - & - & - & D-dimer $\uparrow$, fibrinogen $\uparrow$ & Non-severe \\
\hline de Masson, A. (44) & - & - & - & - & - \\
\hline Freeman EE. (45) & $\begin{array}{l}\text { HTN (2), obstructive lung } \\
\text { disease (2), reumatologic } \\
\text { disease (2) }\end{array}$ & $\begin{array}{l}\text { Fever (9), cough (9), dyspnea } \\
\text { (6), sore throat (5), headache } \\
\text { (7), malaise (4), asymptomatic } \\
\text { (5) }\end{array}$ & - & - & $\begin{array}{l}\text { Outpatient care only (18), Hospitalized } \\
\text { (5), Death (2) }\end{array}$ \\
\hline Le Cleach L. (54) & Raynaud syndrome (2) & $\begin{array}{l}\text { Fever (2), cough (2), dyspnea } \\
(3) \text {, asthenia (5), myalgia (3), } \\
\text { headache (7), odynophagia } \\
\text { (3), anosmia/ageusia (5), } \\
\text { asymptomatic (3) }\end{array}$ & - & - & Outpatient (10) \\
\hline Najarian, D. J. (20) & - & Cough, pain in leg and hands & Azithromycin, Benzonatate & - & Non-severe \\
\hline Sanchez, A. (23) & $\begin{array}{l}\text { T2D, HTN, peripheral } \\
\text { artery disease, chronic } \\
\text { renal failure }\end{array}$ & $\begin{array}{l}\text { Fatigue, fever, dyspnea, acute } \\
\text { respiratory distress }\end{array}$ & Cefpodoxime & $\begin{array}{l}\text { EBV PCR positive (reactivation of } \\
\text { EBV) }\end{array}$ & Severe/ Death \\
\hline Hunt, M. (12) & - & Fever & - & Lymphopenia, CRP $\uparrow$ & Severe \\
\hline Ahouach, B. (25) & - & Fever, dry cough & Paracetamol & - & - \\
\hline Avellana Moreno, R. (34) & - & $\begin{array}{l}\text { Fever, myalgia, asthenia, } \\
\text { cough, diarrhea }\end{array}$ & Paracetamol & - & - \\
\hline Reymundo A. (47) & - & - & - & - & - \\
\hline Morey-Olive, M. (19) & $\begin{array}{l}\text { Cholestatic liver disease } \\
\text { (1) }\end{array}$ & Low-grade fever & Oral symptomatic treatment & $\begin{array}{l}\text { Worsening of the markers for } \\
\text { cholestasis }\end{array}$ & - \\
\hline Gianotti, R. (22) & - & Fever, sore throat, cough & Levofloxacin (3), HCQ (3) & - & Mild (2), Moderate (2), Severe (1) \\
\hline Mahé, A. (16) & T2D & Fever, asthenia, cough & Paracetamol & - & Non-severe/Survived \\
\hline Robustelli Test, E. (42) & - & - & $\begin{array}{l}\text { Lopinavir/ritonavir, HCQ ( } 3 \text { weeks } \\
\text { before) }\end{array}$ & - & - \\
\hline Zengarini, C. (28) & $\begin{array}{l}\text { Moderate obesity, a } \\
\text { history of alcoholism, and } \\
\text { various chronic } \\
\text { morbidities }\end{array}$ & Progressive dyspnoea, fever & $\begin{array}{l}\text { HCQ, Omeprazole, } \\
\text { Piperacillin/Tazobactam, Remdesevir, } \\
\text { Potassium canreonate, and } \\
\text { Enoxaparine. }\end{array}$ & - & Severe \\
\hline Amatore, F. (35) & - & Fever & $\mathrm{HCQ}$ & $\begin{array}{l}\text { Blood count: NL, electrolytes: NL, } \\
\text { CRP: NL, anti-DNA antibodies: NL }\end{array}$ & \\
\hline Gargiulo L. (48) & - & Fever & $\begin{array}{l}\text { Paracetamol, Darunavir/cobicistat, } \\
\mathrm{HCQ}\end{array}$ & - & Severe, Death \\
\hline Ciccarese G. (50) & - & $\begin{array}{l}\text { Fever, sore throat, fatigue, } \\
\text { hyposmia }\end{array}$ & $\begin{array}{l}\text { Cefixime ( } 3 \text { days earlier discontinued), } \\
\text { IVIG, Methylprednisolone }\end{array}$ & $\begin{array}{l}\text { Leukocytosis, Lymphocytosis, severe } \\
\text { Thrombocytopenia, LFT } \uparrow, \mathrm{LDH} \uparrow\end{array}$ & - \\
\hline Recalcati S. (6) & - & - & - & - & - \\
\hline
\end{tabular}




\begin{tabular}{|c|c|c|c|c|c|}
\hline First author & Comorbidity & Associated symptoms & Drug history & Laboratory findings & Severity/outcome \\
\hline Gianotti, R. (21) & - & $\begin{array}{l}\text { Fever (2), cough (2), headache } \\
\text { (1), arthralgias (1) }\end{array}$ & $\begin{array}{l}\text { Lopinavir/Ritonavir (1), Heparin (1), } \\
\text { Levofloxacin (2), Ceftriaxone (1), } \\
\text { Azithromycin (1), HCQ (1) }\end{array}$ & $\mathrm{CRP} \uparrow$, fibrinogen $\uparrow, A L T \uparrow, A S T \uparrow$ & Mild (1), Severe (2) \\
\hline Jimenez-Cauhe J. (41) & - & - & $\begin{array}{l}\text { Lopinavir/Ritonavir, } \mathrm{HCQ} \text {, } \\
\text { Azithromycin, Corticosteroids, } \\
\text { Ceftriaxone }\end{array}$ & $\begin{array}{l}\text { Laboratory tests at the time of skin } \\
\text { lesions showed worsening of one or } \\
\text { more parameters compared to those } \\
\text { at the time of discharge (CRP } \uparrow \\
\text { D-dimer } \uparrow, \text { lymphocyte count } \downarrow \text { ) }\end{array}$ & - \\
\hline Galván Casas C. (24) & - & $\begin{array}{l}\text { Cough, dyspnea, fever, } \\
\text { asthenia, headache, } \\
\text { nausea/vomiting/diarrhea, } \\
\text { anosmia, ageusia, pneumonia }\end{array}$ & - & - & $\begin{array}{l}\text { Pseudo-chilblain: less severe } \\
\text { Vesicular lesions: intermedium } \\
\text { severity } \\
\text { Urticarial and maculopapular lesions: } \\
\text { more severe COVID-19 disease } \\
\text { Livedoid/necrotic lesions: the most } \\
\text { severe disease }\end{array}$ \\
\hline Estébanez, A. (5) & - & $\begin{array}{l}\text { Dry cough, nasal congestion, } \\
\text { fatigue, myalgias, arthralgias, } \\
\text { diarrhea, ageusia, anosmia }\end{array}$ & Paracetamol & - & - \\
\hline Gunawan, C. (43) & $\begin{array}{l}\text { HTN, diabetes, } \\
\text { dyslipidemia, } \\
\text { hyperuricemia }\end{array}$ & $\begin{array}{l}\text { Fever, cough, dyspnea, } \\
\text { diarrhea }\end{array}$ & $\begin{array}{l}\text { Azithromycin, } \mathrm{HCQ} \text {, } \\
\text { Cefoperazone-sulbactam, } \\
\text { Omeprazole, medicines for his } \\
\text { comorbidities }\end{array}$ & - & Non-severe \\
\hline $\begin{array}{l}\text { Quintana-Castanedo, L. } \\
\text { (26) }\end{array}$ & - & $\begin{array}{l}\text { 4-day history of progressive } \\
\text { cutaneous rash }\end{array}$ & - & - & - \\
\hline Henry, D. (30) & - & $\begin{array}{l}\text { Odynophagia, diffuse } \\
\text { arthralgia, chills, chest pain, } \\
\text { fever }\end{array}$ & Paracetamol & Moderate lymphopenia, CRP $\uparrow$ & Non-severe \\
\hline van Damme, C. (33) & $\begin{array}{l}\text { Obesity, T1D, } \\
\text { hypercholesterolemia, } \\
\text { HTN, obstructive sleep } \\
\text { apnea-hypopnea } \\
\text { syndrome, stroke } 18 \\
\text { months ago without } \\
\text { further sequelae, kidney } \\
\text { failure on dialysis }\end{array}$ & General weakness, fever & - & 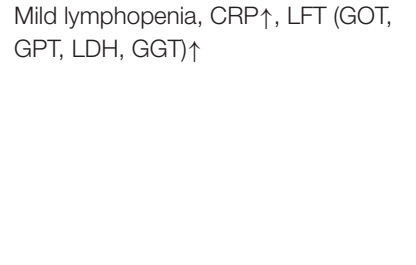 & Severe/Death \\
\hline Paolino, G. (38) & - & $\begin{array}{l}\text { 7th postpartum day, fever, dry } \\
\text { cough, myalgia, arthralgia }\end{array}$ & Paracetamol & - & - \\
\hline Proietti I. (55) & - & Asymptomatic & - & Normal & - \\
\hline Zulfiqar, A. A. (17) & $\begin{array}{l}\text { HTN, autoimmune } \\
\text { hypothyroidism }\end{array}$ & $\begin{array}{l}\text { Fatigue, fever, dry cough, } \\
\text { abdominal discomfort, } \\
\text { epistaxis }\end{array}$ & IV Amoxicillin-Clavulanic acid, LMWH & $\begin{array}{l}\text { CRP } \uparrow, \text { LFT showed cholestasis, } \\
\text { progressive thrombocytopenia, } \\
\text { fibrinogen } \uparrow, \text { TPO } \uparrow\end{array}$ & - \\
\hline Joob, B. (13) & - & $\begin{array}{l}\text { Fever, pneumonia, bleeding } \\
\text { presentation (firstly missed } \\
\text { diagnosed to be dengue) }\end{array}$ & - & - & - \\
\hline
\end{tabular}




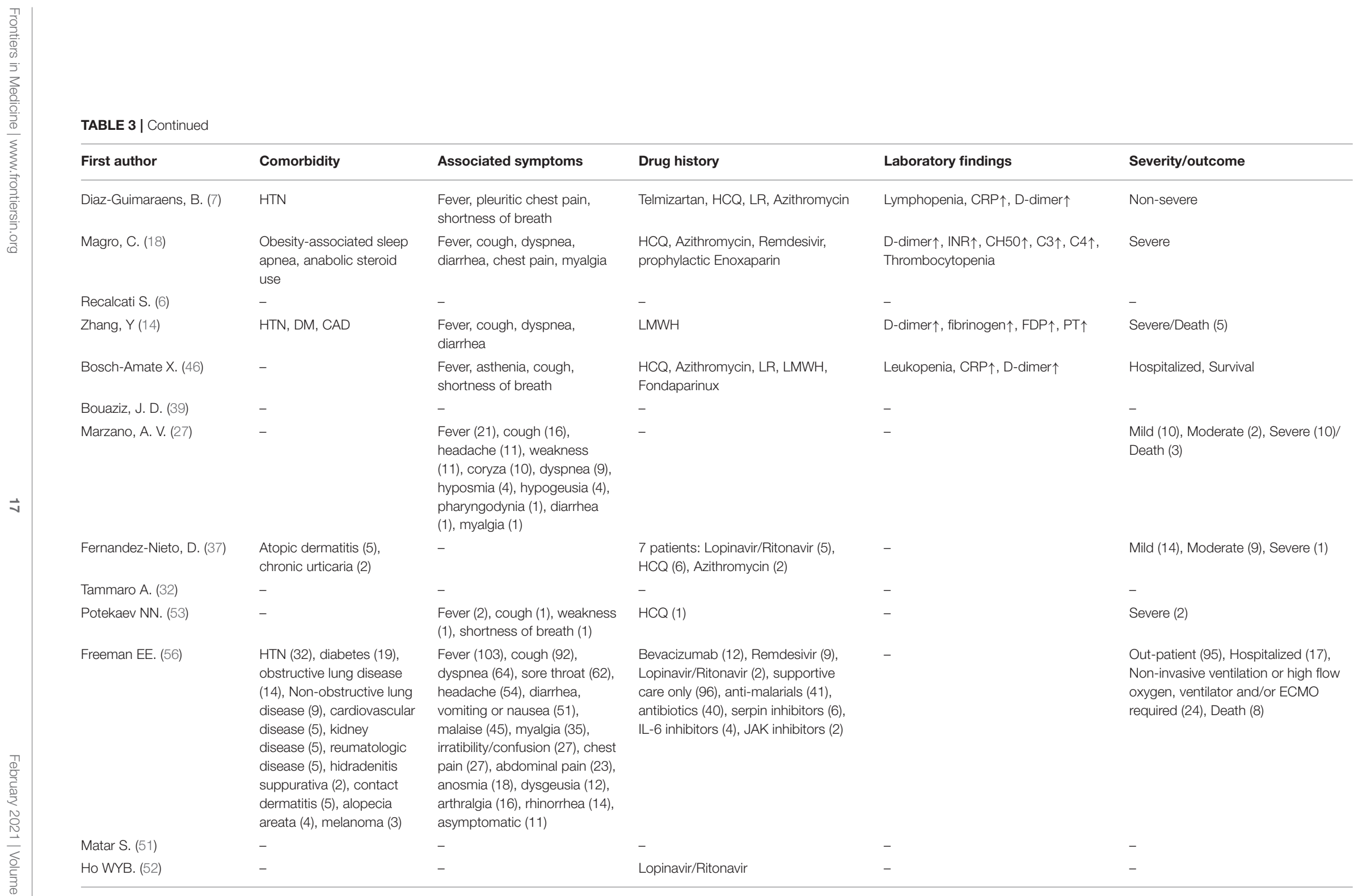

TABLE 3 | Continued 
TABLE 4 | Summary of characteristics of the patients based on the type of lesions.

\begin{tabular}{|c|c|c|c|c|c|c|c|}
\hline Characteristics & $\begin{array}{l}\text { Chilblain-like } \\
\text { lesions }\end{array}$ & $\begin{array}{l}\text { Vesicular } \\
\text { lesions }\end{array}$ & $\begin{array}{l}\text { Urticaria-like } \\
\text { lesions }\end{array}$ & $\begin{array}{l}\text { Maculopapular } \\
\text { lesions }\end{array}$ & $\begin{array}{l}\text { Vascular } \\
\text { lesions }\end{array}$ & Miscellaneous & Total \\
\hline$N(\%)$ & $110(18.4)$ & 89 (15) & 89 (15) & 223 (37.3) & $55(9.2)$ & $31(5.2)$ & 597 \\
\hline Sex, $N^{*}$ & 97 & 83 & 85 & 219 & 46 & 11 & 541 \\
\hline Male, $n(\%)$ & $43(44)$ & $42(51)$ & $28(33)$ & $107(49)$ & $28(61)$ & $2(18)$ & $250(46)$ \\
\hline Female, $n(\%)$ & $54(56)$ & $41(49)$ & $57(67)$ & $112(51)$ & $18(39)$ & $9(82)$ & $291(54)$ \\
\hline Age, mean & 40.7 & 56.1 & 46.3 & 56.4 & 72.3 & 48 & 53.3 \\
\hline $\begin{array}{l}\text { Rash location } \\
\text { Trunk, } n\end{array}$ & 0 & 85 & 91 & 143 & 21 & 23 & 363 \\
\hline Upper Limb, $n$ & 24 & 44 & 29 & 194 & 8 & 9 & 308 \\
\hline Lower Limb, $n$ & 53 & 42 & 30 & 188 & 18 & 10 & 341 \\
\hline Head/Neck, $n$ & 0 & 12 & 20 & 42 & 0 & 7 & 81 \\
\hline Palms/Soles, $n$ & 1 & 2 & 2 & 1 & 1 & 1 & 8 \\
\hline Acral area (Finger, Toe), $n$ & 69 & 0 & 1 & 0 & 18 & 0 & 88 \\
\hline The mucous membrane, $n$ & 0 & 2 & 1 & 4 & 4 & 2 & 13 \\
\hline $\begin{array}{l}\text { Associated cutaneous symptoms, } \\
n(\%)\end{array}$ & $74(67)$ & $62(70)$ & $80(90)$ & $159(71)$ & $18(33)$ & $4(13)$ & $397(66)$ \\
\hline Pruritus, $n$ (\%) & $28(38)$ & $55(89)$ & $22(27.5)$ & $126(79)$ & $4(22)$ & $3(75)$ & $238(60)$ \\
\hline Burning, $n(\%)$ & $40(54)$ & $11(18)$ & $7(9)$ & $23(14)$ & $2(11)$ & $0(0)$ & $83(21)$ \\
\hline Pain, $n$ (\%) & 47 (63.5) & $9(14.5)$ & $6(7.5)$ & $19(27)$ & $3(17)$ & $0(0)$ & $84(21)$ \\
\hline $\begin{array}{l}\text { The onset of the lesions in relation } \\
\text { toother symptoms, } N\end{array}$ & 91 & 86 & 85 & 222 & 41 & 17 & 542 \\
\hline Before, $n(\%)$ & $10(11)$ & $5(6)$ & $5(6)$ & $14(6)$ & $1(2)$ & $1(6)$ & $36(7)$ \\
\hline Chief complaint, $n$ (\%) & $13(14)$ & $0(0)$ & $3(3.5)$ & $3(1)$ & $0(0)$ & $0(0)$ & $19(3.5)$ \\
\hline At the same time, $n(\%)$ & $21(23)$ & $17(20)$ & $40(47)$ & $92(41)$ & $16(39)$ & $1(6)$ & $187(34.5)$ \\
\hline After, $n(\%)$ & $45(49)$ & $64(74)$ & $37(43.5)$ & $113(51)$ & $24(58.5)$ & $15(88)$ & $298(55)$ \\
\hline $\begin{array}{l}\text { Median duration of skin lesions, } \\
\text { day }\end{array}$ & 14 & 9 & 5.25 & 7.4 & 9.5 & 9.3 & 9 \\
\hline Rash treatment, $N^{\star \star}$ & 0 & 0 & 7 & 15 & 5 & 7 & 34 \\
\hline Without treatment, $n(\%)$ & $0(0)$ & $0(0)$ & $0(0)$ & $7(47)$ & $1(20)$ & $0(0)$ & $8(23.5)$ \\
\hline Antihistamines, $n(\%)$ & $0(0)$ & $0(0)$ & $4(57)$ & $0(0)$ & $1(20)$ & $0(0)$ & $5(15)$ \\
\hline Topical corticosteroids, $n$ (\%) & $0(0)$ & $0(0)$ & $1(14)$ & $1(7)$ & $2(40)$ & $1(14)$ & $5(15)$ \\
\hline Systemic corticosteroids, $n$ (\%) & $0(0)$ & $0(0)$ & $2(28.5)$ & $7(47)$ & $2(40)$ & $5(71)$ & $16(47)$ \\
\hline Hydroxychloroquine, $n$ (\%) & $0(0)$ & $0(0)$ & $0(0)$ & $0(0)$ & $0(0)$ & $1(14)$ & $1(3)$ \\
\hline Comrbidity, $N^{* * *}(\%)$ & $20(19)$ & $14(13)$ & $13(12)$ & $43(40)$ & $15(14)$ & $2(2)$ & $107(100)$ \\
\hline Hypertension & $6(30)$ & $3(21)$ & $5(38)$ & $16(37)$ & $12(80)$ & $0(0)$ & $42(39)$ \\
\hline Diabetes & $0(0)$ & $2(14)$ & $6(46)$ & 10(23) & $6(40)$ & $1(50)$ & $25(23)$ \\
\hline Previous dermatologic illness ${ }^{\star \star \star \star}$ & $2(10)$ & $8(57)$ & $1(8)$ & $10(23)$ & $0(0)$ & $0(0)$ & $21(20)$ \\
\hline Obstructive lung disease & $2(10)$ & $1(7)$ & $1(8)$ & $6(14)$ & $6(40)$ & $0(0)$ & $16(15)$ \\
\hline Non-obstructive lung disease & $1(5)$ & $1(7)$ & $3(23)$ & $4(9)$ & $0(0)$ & $0(0)$ & $9(8)$ \\
\hline Rheumatologic disease & $6(30)$ & $1(7)$ & $0(0)$ & $1(2)$ & $1(7)$ & $0(0)$ & $9(8)$ \\
\hline Chronic kidney disease & $0(0)$ & $0(0)$ & $1(8)$ & $6(14)$ & $0(0)$ & $0(0)$ & $7(6.5)$ \\
\hline Cardiovascular disease & $1(5)$ & $0(0)$ & $0(0)$ & $3(7)$ & $2(13)$ & $0(0)$ & $6(6)$ \\
\hline Obesity & $0(0)$ & $0(0)$ & $1(8)$ & $0(0)$ & $1(7)$ & $1(50)$ & $3(3)$ \\
\hline Obstructive sleep apnea & $0(0)$ & $0(0)$ & $1(8)$ & $0(0)$ & $1(7)$ & $0(0)$ & $2(2)$ \\
\hline Dyslipidemia & $0(0)$ & $0(0)$ & $2(15)$ & $0(0)$ & $0(0)$ & $0(0)$ & $2(2)$ \\
\hline Liver disease & $0(0)$ & $0(0)$ & $0(0)$ & $1(2)$ & $0(0)$ & $0(0)$ & $1(1)$ \\
\hline Peripheral artery disease & $0(0)$ & $0(0)$ & $0(0)$ & $1(2)$ & $0(0)$ & $0(0)$ & $1(1)$ \\
\hline Autoimmune hypothyroidism & $0(0)$ & $0(0)$ & $0(0)$ & $0(0)$ & $1(7)$ & $0(0)$ & $1(1)$ \\
\hline Hyperuricemia & $0(0)$ & $0(0)$ & $1(8)$ & $0(0)$ & $0(0)$ & $0(0)$ & $1(1)$ \\
\hline Stroke & $0(0)$ & $0(0)$ & $1(8)$ & $0(0)$ & $0(0)$ & $0(0)$ & $1(1)$ \\
\hline Alcoholism & $0(0)$ & $0(0)$ & $0(0)$ & $0(0)$ & $0(0)$ & $1(50)$ & $1(1)$ \\
\hline
\end{tabular}


TABLE 4 | Continued

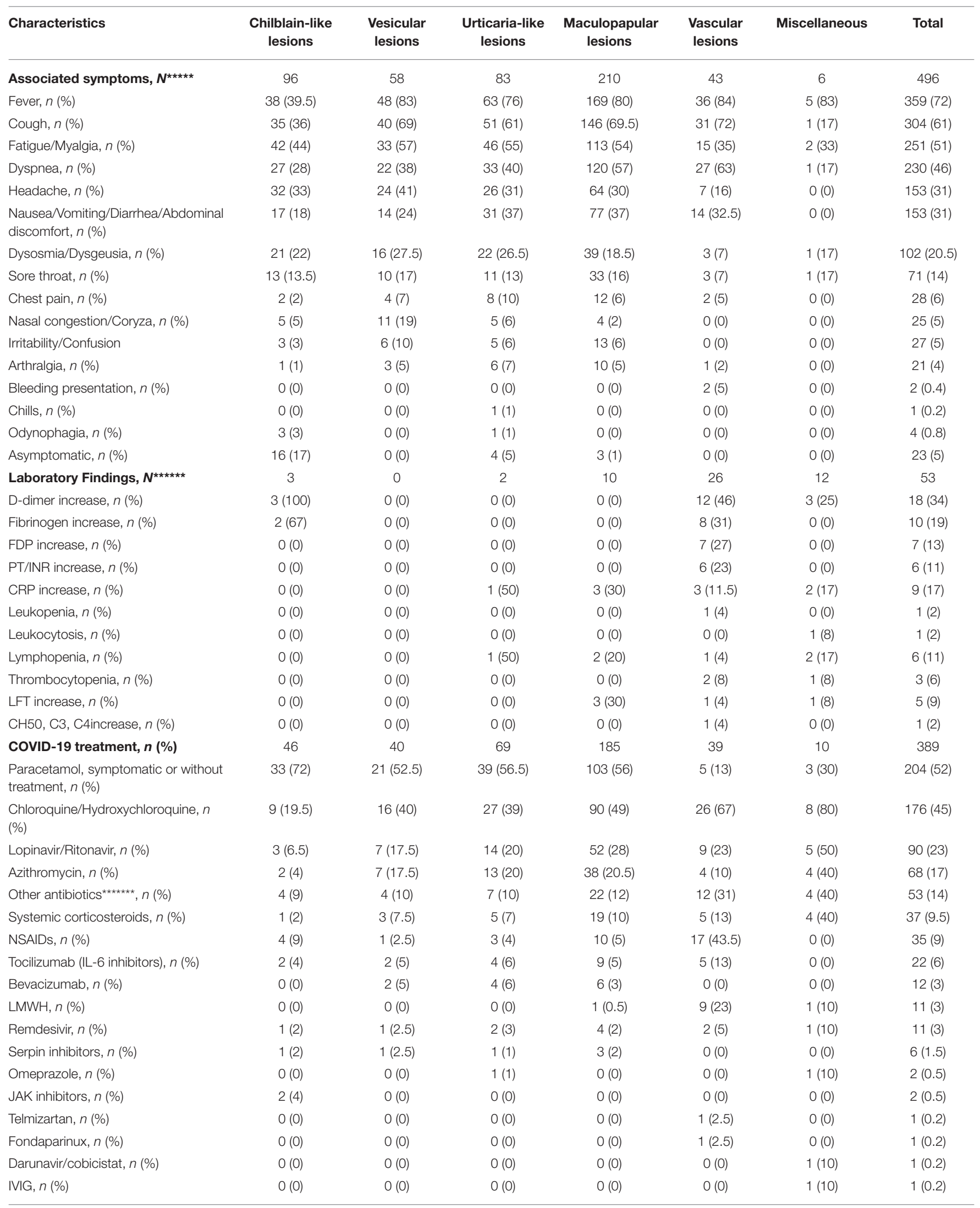


TABLE 4 | Continued

\begin{tabular}{|c|c|c|c|c|c|c|c|}
\hline Characteristics & $\begin{array}{l}\text { Chilblain-like } \\
\text { lesions }\end{array}$ & $\begin{array}{l}\text { Vesicular } \\
\text { lesions }\end{array}$ & $\begin{array}{l}\text { Urticaria-like } \\
\text { lesions }\end{array}$ & $\begin{array}{l}\text { Maculopapular } \\
\text { lesions }\end{array}$ & $\begin{array}{l}\text { Vascular } \\
\text { lesions }\end{array}$ & Miscellaneous & Total \\
\hline COVID-19 severity ${ }^{\star \star \star \star \star \star \star \star *}, N$ & 96 & 63 & 79 & 201 & 44 & 4 & 487 \\
\hline Mild, $n(\%)$ & $79(82)$ & $32(51)$ & $40(51)$ & 79 (39) & $2(5)$ & $2(50)$ & $234(48)$ \\
\hline Moderate, $n(\%)$ & $13(14)$ & $18(29)$ & $28(35)$ & $86(43)$ & $12(27)$ & $0(0)$ & $157(32)$ \\
\hline Severe, $n(\%)$ & $4(4)$ & $13(21)$ & $11(14)$ & $36(18)$ & $30(68)$ & $2(50)$ & $96(20)$ \\
\hline Death, $\boldsymbol{n}(\%)^{\star \star \star \star \star \star \star \star \star ~}$ & $4(3.6)$ & $3(3.4)$ & $2(2.2)$ & $7(3.1)$ & $10(18.2)$ & $1(3.2)$ & $27(4.5)$ \\
\hline
\end{tabular}

FDP, fibrinogen degradation product; PT, prothrombin time; LFT, liver function test.

${ }^{*}$ Number of patients that their gender is reported in the articles.

${ }^{* \star}$ Number of patients that articles mentioned their specific treatment for the lesions.

${ }^{* \star *}$ Number of patients reported having comorbidities in the articles.

${ }^{\star \star \star \star \star}$ E.g., atopic dermatitis, chronic urticaria, melanoma, alopecia areata, hidradenitis suppurativa.

${ }^{\star * \star \star \star}$ Number of patients that articles mentioned their associated symptoms.

${ }^{\star * \star \star \star *}$ Number of patients that their laboratory findings are reported in the articles.

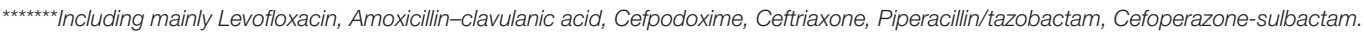

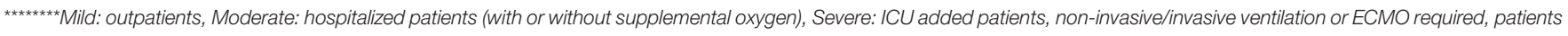
with acute respiratory distress syndrome (ARDS).

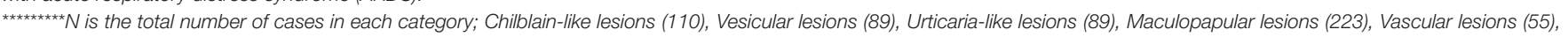
Miscellaneous (31), Total (597).

The overall frequency of cutaneous manifestations in COVID19 patients was $5.95 \%$, with a range from $0.2 \%$ up to $20.4 \%$ in different studies $(6,65)$.

Although skin presentations of COVID-19 are well described, the pathogenesis of skin lesions remains unknown. The direct viral invasion of the skin cells may be one possibility. Angiotensin-converting enzyme 2 (ACE2) is known as a ligand for the Spike protein of SARS-CoV-2 for entering human cells (66). There is a high expression of ACE2 on keratinocytes and sweat gland cells, respectively $(67,68)$. Thus, SARS-CoV-2 can directly infect keratinocytes resulting in necrosis. This hypothesis is consistent with our histologic findings which demonstrated the epidermal and adnexal necrosis in all skin lesions except vesicular rashes. According to Amatore et al., neither viralinduced cytopathic alterations nor intranuclear inclusions were seen in skin biopsies (35). However, SARS-CoV-2 spike and envelope proteins were detected in the endothelial cells of damaged skin in two cases with purpuric rashes (22). RT-PCR for SARS-CoV-2 was performed on skin samples of some patients and was negative in all of them. Since the nasopharyngeal swabs of these patients were positive simultaneously, we assume that it can be a false negative result due to a small viral load or technical problems. Further research is urgently needed.

Skin lesions during SARS-CoV2 infection might be immunerelated phenomena. It has been shown that the presence of virus RNA in blood is related to greater severity of infection (69). Viremia is also associated with the levels of cytokines and growth factors in a dose-dependent manner with markedly higher levels in patients suffering from more severe COVID-19 (69). Recognition of the viral RNA by Toll-free receptors like TLR7 stimulates the intracellular signaling pathways which in turn enhance the cytokine secretion (69).

In a group of patients, with the end of the first week of the infection, a sharp increase in inflammatory cytokines such as interleukin (IL)1, IL2, IL7, IL10, granulocyte colony-stimulating factor (G-CSF), tumor necrosis factor (TNF) $\alpha$ and interferon (IFN)-g occurs. Overactivation of immune responses followed by pro-inflammatory cytokines increase may result in a "cytokine storm" which is an immune pathological condition (69-71). Increased cytokines allow them to access the skin, where they stimulate various cells, including lymphocytes, dendritic cells, macrophages, neutrophils, monocytes, and Langerhans cells to cause various skin manifestations (22, 69). Maybe a hyperviremia state is responsible for vascular lesions in severe COVID-19 patients. We suggest further investigations on the viral load levels among patients with vascular lesions compared with other skin manifestations.

The antigen-antibody complex can lead to complement activation and subsequent mast cell degranulation. This mechanism is suggested particularly for the urticaria-like lesions (43).

A low or delayed interferon response may result in uncontrolled viral replication followed by a subsequent cytokine storm which can lead to severe disease (72). Activation of the host immune system in response to viral antigen deposition may result in vascular damage in COVID-19 infection (73). It seems that high levels of type 1 interferon response, a critical factor in immunity against viral agents, is associated with chilblain-like lesions and mild disease $(15,72,74)$. Activation and aggregation of cytotoxic CD8+ T cells and B cells also lead to lymphocytic thrombophilic arteritis and destruction of keratinocytes $(21,22)$. Nests of Langerhans cells are seen in most of the COVID-19 skin lesion biopsies and have been also reported in another viral-induced skin dermatitis-like pytriasis rosea (75).

Coinfection with other viruses is another potential possibility for COVID-19 related cutaneous manifestations. Some skin lesions in COVID-19 patients are very similar to rashes induced by other viruses like parvovirus18, herpes simplex virus type 1 and 2 (HSV-1, HSV-2), varicella-zoster virus (VZV), and poxviruses, both clinically and histologically. It is probable that because of the attenuation of the immune system, COVID-19 patients are susceptible to coinfection with or relapse of the other viral exanthems. This hypothesis is strongly suggested for vesicular and some miscellaneous lesions (e.g., erythema 
multiform) due to their unique histologic findings compared to other skin lesions of COVID-19 $(24,32,37)$. A study reported four COVID-19 patients presenting diffuse vesicular lesions which microbiological and serological investigations demonstrated varicella infection (24). Thus, in COVID-19 patients with vesicular lesions, physicians need to investigate other possible etiological factors other than SARS-CoV-2.

Coagulopathy and vasculitis are other possible reasons for skin lesions during COVID-19. Evidence shows that COVID19 patients are predisposed to coagulopathy and subsequent thrombotic events (76). It seems to be a result of inflammatory cytokine release, hypoxia, and other illness or therapeutic risk factors (76). Microvascular thrombosis of dermal vessels leads to ischemia or vasculitis mainly seen in chilblain-like or vascular lesions. Magro et al. focused on the role of the complement factors activation, especially alternative and lectin pathways, and subsequent thrombotic microvascular injuries (22). Evidence for this hypothesis is the elevated levels of $\mathrm{CH} 50, \mathrm{C} 3$, and $\mathrm{C} 4$ in blood samples as well as significant vascular depositions of C5b-9, C3d, and C4d in the dermis of skin specimens (22). According to our histologic findings mentioned in RESULT, vascular thrombosis was reported in almost all skin biopsies (except vesicular lesions). This finding across with the increased level of D-dimer, fibrinogen, and prolonged PT and INR in most patients is in favor of this hypothesis. Another presentation of coagulopathy in COVID-19 patients is hemorrhagic events and subsequent dermatologic manifestations (petechiae, purpura, and livedo). These manifestations are not specific to SARS-CoV2. Schneider et al. reported a petechial rash associated with coronavirus NL63 $(77,78)$.

Extremely dilated blood vessels were introduced as a diagnostic histological finding for SARS-CoV-2 by Zengarini et al. (28). There are other reports of vasodilation and telangiectatic vessels in the dermis. With this finding, Magro et al. explained a possible pathway in which dysfunction of ACE2 (due to SARS-CoV-2 binding) and subsequent elevated level of angiotensin2 can result in high activation of endothelial nitric oxide synthase (eNOS) and ensuing vasodilation (22).

Drug-induced eruptions may occur during COVID-19. COVID-19 patients usually use a set of medications that potentially can cause cutaneous rashes. The current study found that paracetamol, azithromycin, hydroxychloroquine, lopinavir/ritonavir, and remdesivir were the most common medications used for COVID-19 patients. Paracetamol has been reported to cause asymmetrical drug-related intertriginous and flexural exanthema (STRIFE) (16). However, in Mahé et al. study, despite keeping the drug, skin lesions disappeared; that is very uncommon in drug reactions (16). Najarian et al. mentioned that maculopapular lesions of their patient could be according to azithromycin use or hypersensitivity reaction to azithromycin due to concurrent viral infection (20).

Hydroxychloroquine that has been used in $45 \%$ of all the cases (mentioned in Result) is one of the most likely medications to cause different skin rashes. Acute generalized exanthematous pustulosis (AGEP), erythroderma, urticaria, and erythema multiform are some of the skin lesions that have been reported in connection with hydroxychloroquine (79-81).
However, Robustelli et al. mentioned that the skin lesion developed 3 weeks after discontinuation of the drug (42). As a conclusion, most of our reviewed articles considered the potential possibility of drug-induced exanthems but in almost all cases, dermatologic manifestations preceded the drug intake or the rashes disappeared despite the continuation of drugs $(5,7,16,20,23,37,42,43)$. So it is very unlikely that current COVID-19 medications are responsible for the reported skin lesions.

In our study, the prevalence of comorbidities in COVID19 patients with skin manifestations is about $17.9 \%$ mainly reported in patients with maculopapular lesions. History of serious comorbidities like cardiovascular disease, hypertension, and obstructive lung disease was mostly reported in patients with vascular lesions; suggesting that patients with these skin manifestations are more complicated cases and need more attention. Interestingly, immune disorders were more common in patients with chilblain-like lesions. This finding is not reported yet and we suggest it to be focused on due to the possible relationship with the etiology and pathophysiology of these lesions.

Fever, cough, and dyspnea were more frequent in patients with vascular lesions and less frequent in patients with chilblainlike lesions. Also, $17 \%$ of patients with chilblain-like lesions were asymptomatic regarding systemic symptoms. Astonishingly, headache, dysosmia/dysgeusia, nasal congestion/coryza, and irritability/confusion were more common in patients with vesicular lesions. This finding can demonstrate the probable link between vesicular lesions and neurological manifestations. Future investigations are required to clarify the issue.

\section{LIMITATIONS}

There were limited articles that mentioned complete data about all the items including the disease severity and outcome of the COVID-19 patients with dermatologic presentations. Another limitation was the absence of data about the COVID-19 patients without skin manifestations. Future cohort studies are required to compare the severity and prognosis of the disease in patients with and without skin manifestations, considering other related characteristics. Such studies help to better understand the prognostic value of the cutaneous manifestations in COVID19 patients.

\section{CONCLUSIONS}

Cutaneous lesions occur most often in middle age individuals at the same time or after the systemic symptoms of COVID-19. Urticaria-like lesions commonly (47\%) occurred at the same time with other symptoms. It may suggest that urticaria-like lesions may be a diagnostic sign for COVID-19. A maculopapular rash is the main reported skin involvement in COVID-19 patients and is associated with intermediate severity of the disease. The mere occurrence of skin manifestations in COVID-19 patients is not an indicator for the disease severity, and it highly 
depends on the type of skin lesions. Chilblain-like and vascular lesions are the ends of a spectrum in which from chilblain-like to vascular lesions, the severity of the disease increases, and the patient's prognosis worsens. We highly suggest emergency and general practitioners to evaluate the suspected COVID-19 patients for any cutaneous manifestations. Those with vascular lesions should also be considered as high-priority patients for further medical care.

\section{DATA AVAILABILITY STATEMENT}

The original contributions presented in the study are included in the article/supplementary material, further inquiries can be directed to the corresponding author/s.

\section{REFERENCES}

1. Zhu N, Zhang D, Wang W, Li X, Yang B, Song J, et al. A novel coronavirus from patients with pneumonia in China, 2019. N Engl J Med. (2020) 382:72733. doi: 10.1056/NEJMoa2001017

2. World Health Organization. Coronavirus Disease 2019 (COVID-19): Situation Report, 88. Geneva: World Health Organization (2020).

3. Tahvildari A, Arbabi M, Farsi Y, Jamshidi P, Hasanzadeh S, Calcagno TM, et al. Clinical features, diagnosis, and treatment of COVID-19 in hospitalized patients: a systematic review of case reports and case series. Front Med. (2020) 7:231. doi: 10.3389/fmed.2020.00231

4. Giavedoni P, Podlipnik S, Pericàs JM, Fuertes de Vega I, García-Herrera A, Alós L, et al. Skin manifestations in COVID-19: prevalence and relationship with disease severity. J Clin Med. (2020) 9:3261. doi: 10.3390/jcm9103261

5. Estébanez A, Pérez-Santiago L, Silva E, Guillen-Climent S, García-Vázquez A, Ramón MD. Cutaneous manifestations in COVID-19: a new contribution. $J$ Eur Acad Dermatol Venereol. (2020) 34:e250-1. doi: 10.1111/jdv.16474

6. Recalcati S. Cutaneous manifestations in COVID-19: a first perspective. J Eur Acad Dermatol Venereol. (2020) 34:e212-3. doi: 10.1111/jdv.16387

7. Diaz-Guimaraens B, Dominguez-Santas M, Suarez-Valle A, Pindado-Ortega C, Selda-Enriquez G, Bea-Ardebol S, et al. Petechial skin rash associated with severe acute respiratory syndrome coronavirus 2 infection. JAMA Dermatol. (2020) 156:820-2. doi: 10.1001/jamadermatol.2020.1741

8. Piccolo V, Neri I, Filippeschi C, Oranges T, Argenziano G, Battarra VC, et al. Chilblain-like lesions during COVID-19 epidemic: a preliminary study on 63 patients. J Eur Acad Dermatol Venereol. (2020) 34:e291-3. doi: $10.1111 /$ jdv.16526

9. Young S, Fernandez AP. Skin manifestations of COVID-19. Cleveland Clin J Med. (2020). doi: 10.3949/ccjm.87a.ccc031. [Epub ahead of print].

10. Moher D, Liberati A, Tetzlaff J, Altman DG. Preferred reporting items for systematic reviews and meta-analyses: the PRISMA statement. Ann Intern Med. (2009) 151:264-9. doi: 10.7326/0003-4819-151-4-200908180-00135

11. The Joanna Briggs Institute. The Joanna Briggs Institute Critical Appraisal Tools for Use in JBI Systematic Review: Checklists for Case Reports. Adelaide: The Joanna Briggs Institute (2019).

12. Hunt M, Koziatek C. A case of COVID-19 pneumonia in a young male with full body rash as a presenting symptom. Clin Pract Cases Emerg Med. (2020) 4:219-21. doi: 10.5811/cpcem.2020.3.47349

13. Joob B, Wiwanitkit V. Hemorrhagic problem among the patients with COVID-19: clinical summary of 41 thai infected patients. Clin Appl Thromb Hemost. (2020) 26:1076029620918308. doi: 10.1177/1076029620918308

14. Zhang Y, Cao W, Xiao M, Li YJ, Yang Y, Zhao J, et al. Clinical and coagulation characteristics of 7 patients with critical COVID-2019 pneumonia and acro-ischemia. Zhonghua xue ye xue za zhi. (2020) 41:E006. doi: 10.3760/cma.j.issn.0253-2727.2020.0006

15. Fiehn C. Familial chilblain lupus-what can we learn from type I interferonopathies? Curr Rheumatol Rep. (2017) 19:61. doi: 10.1007/s11926-017-0689-x

\section{AUTHOR CONTRIBUTIONS}

PJ, MN, and MM designed the study. PJ and $\mathrm{BH}$ performed the review literatures, collected the data, and wrote the first draft of the manuscript. PJ, HV, and MD helped in manuscript preparation. MM critically reviewed the manuscript.

\section{ACKNOWLEDGMENTS}

This study was related to the project No. 25240 From Student Research Committee, Shahid Beheshti University of Medical Sciences, Tehran, Iran. We also appreciate Student Research Committee and Research and Technology Chancellor in Shahid Beheshti University of Medical Sciences for their financial support of this study.

16. Mahé A, Birckel E, Krieger S, Merklen C, Bottlaender L. A distinctive skin rash associated with coronavirus disease 2019? J Eur Acad Dermatol Venereol. (2020) 34:e246-7. doi: 10.1111/jdv.16471

17. Zulfiqar AA, Lorenzo-Villalba $\mathrm{N}$, Hassler $\mathrm{P}$, Andres E. Immune thrombocytopenic purpura in a patient with Covid-19. N Engl J Med. (2020) 382:e43. doi: 10.1056/NEJMc2010472

18. Magro C, Mulvey JJ, Berlin D, Nuovo G, Salvatore S, Harp J, et al. Complement associated microvascular injury and thrombosis in the pathogenesis of severe COVID-19 infection: a report of five cases. Transl Res. (2020) 220:1-13. doi: $10.1016 /$ j.trsl.2020.04.007

19. Morey-Olive M, Espiau M, Mercadal-Hally M, Lera-Carballo E, Garcia-Patos V. Cutaneous manifestations in the current pandemic of coronavirus infection disease (COVID 2019). Anal Pediatr. (2020) 92:374-5. doi: 10.1016/j.anpede.2020.04.002

20. Najarian DJ. Morbilliform exanthem associated with COVID-19. JAAD Case Rep. (2020) 6:493. doi: 10.1016/j.jdcr.2020.04.015

21. Gianotti R, Veraldi S, Recalcati S, Cusini M, Ghislanzoni M, Boggio F, et al. Cutaneous clinico-pathological findings in three COVID-19-positive patients observed in the metropolitan area of Milan, Italy. Acta Dermatovenereol. (2020) 100:1-2. doi: 10.2340/00015555-3490

22. Gianotti R, Zerbi P, Dodiuk-Gad RP. Clinical and histopathological study of skin dermatoses in patients affected by COVID-19 infection in the Northern part of Italy. J Dermatol Sci. (2020) 98:141-3. doi: 10.1016/j.jdermsci.2020.04.007

23. Sanchez A, Sohier P, Benghanem S, L'Honneur AS, Rozenberg F, Dupin $\mathrm{N}$, et al. Digitate papulosquamous eruption associated with severe acute respiratory syndrome coronavirus 2 infection. JAMA Dermatol. (2020) 156:819-20. doi: 10.1001/jamadermatol.2020.1704

24. Galván Casas C, Catala A, Carretero Hernández G, Rodríguez-Jiménez P, Fernández-Nieto D, Rodríguez-Villa Lario A, et al. Classification of the cutaneous manifestations of COVID-19: a rapid prospective nationwide consensus study in Spain with 375 cases. Br J Dermatol. (2020) 183:71-7. doi: 10.1111/bjd.19163

25. Ahouach B, Harant S, Ullmer A, Martres P, Begon E, Blum L, et al. Cutaneous lesions in a patient with COVID-19: are they related? Br J Dermatol. (2020) 183:e31. doi: 10.1111/bjd.19168

26. Quintana-Castanedo L, Feito-Rodriguez M, Valero-Lopez I, ChiloechesFernandez C, Sendagorta-Cudos E, Herranz-Pinto P. Urticarial exanthem as early diagnostic clue for COVID-19 infection. JAAD Case Rep. (2020) 6:498-9. doi: $10.1016 /$ j.jdcr.2020.04.026

27. Marzano AV, Genovese G, Fabbrocini G, Pigatto P, Monfrecola G, Piraccini $\mathrm{BM}$, et al. Varicella-like exanthem as a specific COVID-19-associated skin manifestation: multicenter case series of 22 patients. J Am Acad Dermatol. (2020) 83:280-5. doi: 10.1016/j.jaad.2020.04.044

28. Zengarini C, Orioni G, Cascavilla A, Horna Solera C, Fulgaro C, Misciali C, et al. Histological pattern in Covid-19 induced viral rash. J Eur Acad Dermatol Venereol. (2020) 34:e453-4. doi: 10.1111/jdv. 16569 
29. Alramthan A, Aldaraji W. A case of COVID-19 presenting in clinical picture resembling chilblains disease. First report from the Middle East. Clin Exp Dermatol. (2020) 45:746-8. doi: 10.1111/ced.14243

30. Henry D, Ackerman M, Sancelme E, Finon A, Esteve E. Urticarial eruption in COVID-19 infection. J Eur Acad Dermatol Venereol. (2020) 34:e244-5. doi: $10.1111 /$ jdv. 16472

31. Recalcati S, Barbagallo T, Frasin L, Prestinari F, Cogliardi A, Provero M, et al. Acral cutaneous lesions in the time of COVID-19. J Eur Acad Dermatol Venereol. (2020) 34:e346-7. doi: 10.1111/jdv.16533

32. Tammaro A, Adebanjo GAR, Parisella FR, Pezzuto A, Rello J. Cutaneous manifestations in COVID-19: the experiences of Barcelona and Rome. J Eur Acad Dermatol Venereol. (2020) 34:e306-7. doi: 10.1111/jdv.16530

33. van Damme C, Berlingin E, Saussez S, Accaputo O. Acute urticaria with pyrexia as the first manifestations of a COVID-19 infection. $J$ Eur Acad Dermatol Venereol. (2020) 34:e300-1. doi: 10.1111/jdv.16523

34. Avellana Moreno R, Villa E, Avellana Moreno V, Estela Villa C, Aparicio M, Fontanella A. Cutaneous manifestation of COVID-19 in images: a case report. J Eur Acad Dermatol Venereol. (2020) 34:e307-9. doi: 10.1111/jdv.16531

35. Amatore F, Macagno N, Mailhe M, Demarez B, Gaudy-Marqueste C, Grob JJ, et al. SARS-CoV-2 infection presenting as a febrile rash. J Eur Acad Dermatol Venereol. (2020) 34:e304-6. doi: 10.1111/jdv.16528

36. Suarez-Valle A, Fernandez-Nieto D, Diaz-Guimaraens B, DominguezSantas M, Carretero I, Perez-Garcia B. Acro-ischemia in hospitalized COVID-19 patients. J Eur Acad Dermatol Venereol. (2020) 34:e455-7. doi: $10.1111 /$ jdv. 16592

37. Fernandez-Nieto D, Ortega-Quijano D, Jimenez-Cauhe J, BurgosBlasco P, de Perosanz-Lobo D, Suarez-Valle A, et al. Clinical and histological characterization of vesicular COVID-19 rashes: a prospective study in a tertiary care hospital. Clin Exp Dermatol. (2020) 45:872-5. doi: $10.1111 /$ ced.14277

38. Paolino G, Canti V, Raffaele Mercuri S, Rovere Querini P, Candiani M, Pasi F. Diffuse cutaneous manifestation in a new mother with COVID-19 (SARS-Cov-2). Int J Dermatol. (2020) 59:874-5. doi: 10.1111/ijd.14919

39. Bouaziz JD, Duong T, Jachiet M, Velter C, Lestang P, Cassius C, et al. Vascular skin symptoms in COVID-19: a french observational study. J Eur Acad Dermatol Venereol. (2020) 34:e451-2. doi: 10.1111/jdv.16544

40. Locatelli AG, Robustelli Test E, Vezzoli P, Carugno A, Moggio E, Consonni $\mathrm{L}$, et al. Histologic features of long lasting chilblain-like lesions in a pediatric COVID-19 patient. J Eur Acad Dermatol Venereol. (2020) 34:e365-8. doi: $10.1111 /$ jdv.16617

41. Jimenez-Cauhe J, Ortega-Quijano D, Carretero-Barrio I, Suarez-Valle A, Saceda-Corralo D, Moreno-Garcia Del Real C, et al. Erythema multiformelike eruption in patients with COVID-19 infection: clinical and histological findings. Clin Exp Dermatol. (2020) 45:892-5. doi: 10.1111/ced.14281

42. Robustelli Test E, Vezzoli P, Carugno A, Raponi F, Gianatti A, Rongioletti F, et al. Acute generalized exanthematous pustulosis with erythema multiformelike lesions in a COVID-19 woman. J Eur Acad Dermatol Venereol. (2020) 34:e457-9. doi: 10.1111/jdv.16613

43. Gunawan C, Angela, Widysanto A. Urticarial eruption in coronavirus disease 2019 (COVID-19) infection: a case report in Tangerang, Indonesia. J Eur Acad Dermatol Venereol. (2020) 34:e372-3. doi: 10.1111/jdv.16622

44. de Masson A, Bouaziz JD, Sulimovic L, Cassius C, Jachiet M, Ionescu MA, et al. Chilblains are a common cutaneous finding during the COVID-19 pandemic: a retrospective nationwide study from France. J Am Acad Dermatol. (2020) 83:667-70. doi: 10.1016/j.jaad.2020.04.161

45. Freeman EE, McMahon DE, Lipoff JB, Rosenbach M, Kovarik C, Takeshita J, et al. Pernio-like skin lesions associated with COVID-19: a case series of 318 patients from 8 countries. J Am Acad Dermatol. (2020) 83:486-92. doi: 10.1016/j.jaad.2020.05.109

46. Bosch-Amate X, Giavedoni P, Podlipnik S, Andreu-Febrer C, Sanz-Beltran J, Garcia-Herrera A, et al. Retiform purpura as a dermatological sign of covid-19 coagulopathy. J Eur Acad Dermatol Venereol. (2020) 34:e548-9. doi: $10.1111 /$ jdv.16689

47. Reymundo A, Fernáldez-Bernáldez A, Reolid A, Butrón B, Fernández-Rico P, Muñoz-Hernández P, et al. Clinical and histological characterization of late appearance maculopapular eruptions in association with the coronavirus disease 2019. A case series of seven patients. J Eur Acad Dermatol Venereol. (2020) 34:e755-7. doi: 10.1111/jdv.16707
48. Gargiulo L, Pavia G, Facheris P, Valenti M, Sacrini F, Narcisi A, et al. A fatal case of COVID-19 infection presenting with an erythema multiformelike eruption and fever. Dermatol Ther. (2020) 33:e13779. doi: 10.1111/dth. 13779

49. Askin O, Altunkalem RN, Altinisik DD, Uzuncakmak TK, Tursen U, Kutlubay Z. Cutaneous manifestations in hospitalized patients diagnosed as COVID-19. Dermatol Ther. (2020) 33:e13896. doi: 10.1111/dth.13896

50. Ciccarese G, Drago F, Boatti M, Porro A, Muzic SI, Parodi A. Oral erosions and petechiae during SARS-CoV-2 infection. J Med Virol. (2021) 93:129-32. doi: $10.1002 /$ jmv. 26221

51. Matar S, Oulès B, Sohier P, Chosidow O, Beylot-Barry M, Dupin N, et al. Cutaneous manifestations in SARS-CoV-2 infection (COVID-19): a French experience and a systematic review of the literature. J Eur Acad Dermatol Venereol. (2020) 34:e686-9. doi: 10.1111/jdv.16775

52. Ho BWY, Wang D, Tan LYC, Bundele MM, Tan CH, Lim JHL. Two cases of cutaneous eruptions due to CoVID-19 infection in Singapore: new insights into the spectrum of clinical presentation and histopathology. J Eur Acad Dermatol Venereol. (2020) 34:e532-652. doi: 10.1111/jdv.16736

53. Potekaev NN, Zhukova OV, Protsenko DN, Demina OM, Khlystova EA, Bogin V. Clinical characteristics of dermatologic manifestations of COVID19 infection: case series of 15 patients, review of literature, and proposed etiological classification. Int J Dermatol. (2020) 59:1000. doi: 10.1111/ijd. 15030

54. Le Cleach L, Dousset L, Assier H, Fourati S, Barbarot S, Boulard C, et al. Most chilblains observed during the COVID-19 outbreak occur in patients who are negative for COVID-19 on PCR and serology testing. Br J Dermatol. (2020) 183:866-74. doi: 10.1111/bjd.19377

55. Proietti I, Mambrin A, Bernardini N, Tolino E, Balduzzi V, Maddalena P, et al. Urticaria in an infant with SARS-CoV-2 positivity. Dermatol Ther. (2020) 33:e14043. doi: 10.1111/dth.14043

56. Freeman EE, McMahon DE, Lipoff JB, Rosenbach M, Kovarik C, Desai SR, et al. The spectrum of COVID-19-associated dermatologic manifestations: An international registry of 716 patients from 31 countries. J Am Acad Dermatol. (2020) 83:1118-29. doi: 10.1016/j.jaad.2020.06.1016

57. Gandhi M, Yokoe DS, Havlir DV. Asymptomatic transmission, the Achilles' heel of current strategies to control COVID-19. Mass Med Soc. (2020) 382:2158-60. doi: 10.1056/NEJMe2009758

58. Arons MM, Hatfield KM, Reddy SC, Kimball A, James A, Jacobs JR, et al. Presymptomatic SARS-CoV-2 infections and transmission in a skilled nursing facility. N Engl J Med. (2020) 382:2081-90. doi: 10.1056/NEJMoa20 08457

59. Lu S, Lin J, Zhang Z, Xiao L, Jiang Z, Chen J, et al. Alert for non-respiratory symptoms of coronavirus disease 2019 (COVID-19) patients in epidemic period: a case report of familial cluster with three asymptomatic COVID-19 patients. J Med Virol. (2020) 93:518-21. doi: 10.1002/jmv.25776

60. Black JR, Bailey C, Przewrocka J, Dijkstra KK, Swanton C. COVID-19: the case for health-care worker screening to prevent hospital transmission. Lancet. (2020) 395:1418-20. doi: 10.1016/S0140-6736(20)30917-X

61. Hu Y, Sun J, Dai Z, Deng H, Li X, Huang Q, et al. Prevalence and severity of corona virus disease 2019 (COVID-19): a systematic review and metaanalysis. J Clin Virol. (2020) 127:104371. doi: 10.1016/j.jcv.2020.104371

62. Nasiri MJ, Haddadi S, Tahvildari A, Farsi Y, Arbabi M, Hasanzadeh $\mathrm{S}$, et al. COVID-19 clinical characteristics, and sex-specific risk of mortality: systematic review and meta-analysis. Front Med. (2020) 7:459. doi: $10.3389 /$ fmed.2020.00459

63. Wu Z, McGoogan JM. Characteristics of and important lessons from the coronavirus disease 2019 (COVID-19) outbreak in China: summary of a report of 72314 cases from the Chinese Center for Disease Control and Prevention. JAMA. (2020) 323:1239-42. doi: 10.1001/jama.2020.2648

64. Gupta MK, Lipner SR. Personal protective equipment recommendations based on COVID-19 route of transmission. J Am Acad Dermatol. (2020) 83:e45-6. doi: 10.1016/j.jaad.2020.04.068

65. Guan WJ, Ni ZY, Hu Y, Liang WH, Ou CQ, He JX, et al. Clinical characteristics of coronavirus disease 2019 in China. N Engl J Med. (2020) 382:1708-20. doi: 10.1056/NEJMoa2002032

66. Walls AC, Park YJ, Tortorici MA, Wall A, McGuire AT, Veesler D. Structure, function, and antigenicity of the SARS-CoV-2 spike glycoprotein. Cell. (2020) 181:281-92.e6. doi: 10.1016/j.cell.2020.11.032 
67. Xue X, Mi Z, Wang Z, Pang Z, Liu H, Zhang F. High expression of ACE2 on keratinocytes reveals skin as a potential target for SARS-CoV-2. J Investig Dermatol. (2021) 141:206-9.e1. doi: 10.1016/j.jid.2020.05.087

68. Li MY, Li L, Zhang Y, Wang XS. Expression of the SARS-CoV-2 cell receptor gene ACE2 in a wide variety of human tissues. Infect Dis Poverty. (2020) 9:45. doi: 10.1186/s40249-020-00662-X

69. Li G, Fan Y, Lai Y, Han T, Li Z, Zhou P, et al. Coronavirus infections and immune responses. J Med Virol. (2020) 92:424-32. doi: 10.1002/jmv.25685

70. Singhal T. A review of coronavirus disease-2019 (COVID-19). Indian J Pediatr. (2020) 92:424-32. doi: 10.1007/s12098-020-03263-6

71. Chen Y, Liu Q, Guo D. Emerging coronaviruses: genome structure, replication, and pathogenesis. J Med Virol. (2020) 92:418-23. doi: 10.1002/jmv.26234

72. Jamilloux Y, Henry T, Belot A, Viel S, Fauter M, El Jammal T, et al. Should we stimulate or suppress immune responses in COVID-19? Cytokine and anti-cytokine interventions. Autoimmun Rev. (2020) 19:102567. doi: 10.1016/j.autrev.2020.102567

73. Mayor-Ibarguren A, Feito-Rodriguez M, Quintana Castanedo L, Ruiz-Bravo E, Montero Vega D, Herranz-Pinto P. Cutaneous small vessel vasculitis secondary to COVID-19 infection: a case report. J Eur Acad Dermatol Venereol. (2020) 34:e541-2. doi: 10.1111/jdv.16670

74. Damsky W, Peterson D, King B. When interferon tiptoes through COVID-19: pernio-like lesions and their prognostic implications during SARS-CoV-2 infection. J Am Acad Dermatol. (2020) 83:e269-70. doi: 10.1016/j.jaad.2020.06.052

75. Broccolo F, Ciccarese G, Oggioni M, Rebora A, Parodi A, Drago F. The saliva quantitative PCR assay is inadequate to detect and monitor human herpesvirus-7 and -6 reactivation in patients with Pityriasis rosea. J Clin Virol. (2014) 61:615-6. doi: 10.1016/j.jcv.2014.09.020

76. Bikdeli B, Madhavan MV, Jimenez D, Chuich T, Dreyfus I, Driggin E, et al. COVID-19 and thrombotic or thromboembolic disease: implications for prevention, antithrombotic therapy, and follow-up: JACC state-of-the-art review. J Am Coll Cardiol. (2020) 75:2950-73. doi: 10.1016/j.jacc.2020.04.031

77. Schneider H, Adams O, Weiss C, Merz U, Schroten H, Tenenbaum T. Clinical characteristics of children with viral single- and coinfections and a petechial rash. Pediatr infect Dis J. (2013) 32:e186-91. doi: 10.1097/INF.0b013e318280618d

78. Chesser H, Chambliss JM, Zwemer E. Acute hemorrhagic edema of infancy after coronavirus infection with recurrent rash. Case Rep Pediatr. (2017) 2017:5637503. doi: 10.1155/2017/5637503

79. Abou Assalie N, Durcan R, Durcan L, Petri MA. Hydroxychloroquineinduced erythema multiforme. J Clin Rheumatol. (2017) 23:127-8. doi: 10.1097/RHU.0000000000000417

80. Rodriguez-Caruncho C, Bielsa Marsol I. Antimalarials in dermatology: mechanism of action, indications, and side effects. Actas Dermsifiliogr. (2014) 105:243-52. doi: 10.1016/j.adengl.2012.10.021

81. Liccioli G, Marrani E, Giani T, Simonini G, Barni S, Mori F. The first pediatric case of acute generalized exanthematous pustulosis caused by hydroxychloroquine. Pharmacology. (2019) 104:57-9. doi: 10.1159/0005 00406

Conflict of Interest: The authors declare that the research was conducted in the absence of any commercial or financial relationships that could be construed as a potential conflict of interest.

Copyright (c) 2021 Jamshidi, Hajikhani, Mirsaeidi, Vahidnezhad, Dadashi and Nasiri. This is an open-access article distributed under the terms of the Creative Commons Attribution License (CC BY). The use, distribution or reproduction in other forums is permitted, provided the original author(s) and the copyright owner(s) are credited and that the original publication in this journal is cited, in accordance with accepted academic practice. No use, distribution or reproduction is permitted which does not comply with these terms. 